San Jose State University

SJSU ScholarWorks

Master's Theses

Master's Theses and Graduate Research

Spring 2010

\title{
Surface Plasmon Studies of Composition Control in Mixed Acid Alkanethiol SAMs
}

Arthur Cheng

San Jose State University

Follow this and additional works at: https://scholarworks.sjsu.edu/etd_theses

\section{Recommended Citation}

Cheng, Arthur, "Surface Plasmon Studies of Composition Control in Mixed Acid Alkanethiol SAMs" (2010). Master's Theses. 3753.

DOI: https://doi.org/10.31979/etd.zwcj-x9ab

https://scholarworks.sjsu.edu/etd_theses/3753

This Thesis is brought to you for free and open access by the Master's Theses and Graduate Research at SJSU ScholarWorks. It has been accepted for inclusion in Master's Theses by an authorized administrator of SJSU ScholarWorks. For more information, please contact scholarworks@sjsu.edu. 


\title{
SURFACE PLASMON STUDIES OF COMPOSITION CONTROL IN MIXED ACID ALKANETHIOL SAMs
}

\author{
A Thesis \\ Presented to \\ The Faculty of the Department of Chemistry \\ San José State University \\ In Partial Fulfillment \\ of the Requirements for the Degree \\ Master of Science
}

by

Arthur Cheng

May 2010 
(C) 2010

Arthur Cheng

ALL RIGHTS RESERVED 
The Designated Thesis Committee Approves the Thesis Titled

SURFACE PLASMON STUDIES OF COMPOSITIONAL CONTROL IN MIXED ACID ALKANETHIOL SAMS

by

Arthur Cheng

\begin{abstract}
APPROVED FOR THE DEPARTMENT OF CHEMISTRY
SAN JOSÉ STATE UNIVERSITY
\end{abstract}

May 2010

Dr. Roger Terrill Department of Chemistry

Dr. Daryl Eggers Department of Chemistry

Dr. Joseph Pesek Department of Chemistry 


\section{ABSTRACT \\ SURFACE PLASMON STUDIES OF COMPOSITIONAL CONTROL IN MIXED ACID ALKANETHIOL SAMS \\ by Arthur Cheng}

In this study, surface plasmon resonance (SPR) was used to investigate the adsorption of MUA (11-mercapto-undecanoic acid), MPA (3-mercapto-propionic acid) and MHX (6-mercapto-1-hexanol) onto a gold SPR sensor surface. MUA, MPA, and MHX were dissolved in phosphate buffers of ionic strengths varying from $0.1 \mathrm{mM}$ to $1 \mathrm{M}$ and at $\mathrm{pH}$ values between 2 and 11, and monolayer formation experiments were performed. Our results illustrate how the adsorption of these charged and neutral alkanethiols depends on solution ionic strength and $\mathrm{pH}$. Results indicate that, for single-component MUA or MPA layers, low $\mathrm{pH}$ and elevated solution ionic strength may promote denser layer formation. For binary MUA-MHX monolayers, potentiostatic control was important to establish reproducible SAM formation. Surprisingly, for $0.00 \mathrm{~V}$ potentiostatic depositions from mixed MUA-MHX solutions, MUA was substantially incorporated into the monolayer only at $\mathrm{pH} 3$; whereas, at $\mathrm{pH} 7$ and $11, \mathrm{MHX}$ strongly predominated in the monolayer phase. 


\section{TABLE OF CONTENTS}

LIST OF FIGURES

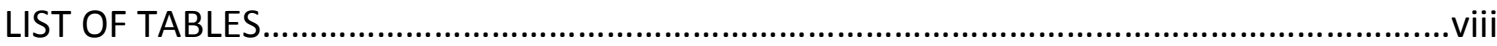

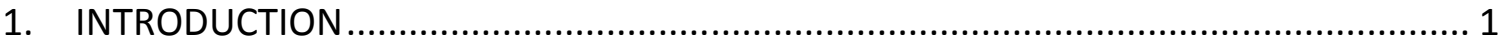

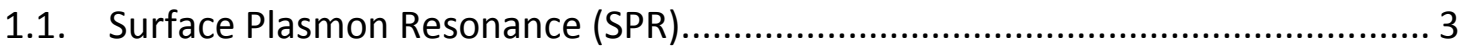

1.2. Use of Surface Plasmon Resonance (SPR) as a Biosensor ................................ 6

1.3. Self Assembled Monolayer (SAM) Formation ............................................... 7

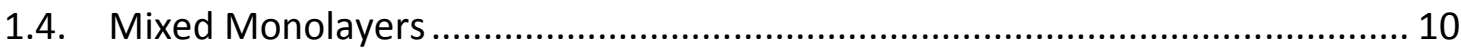

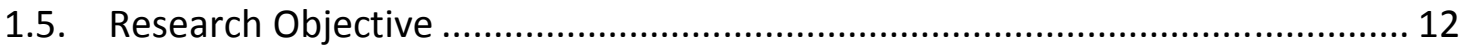

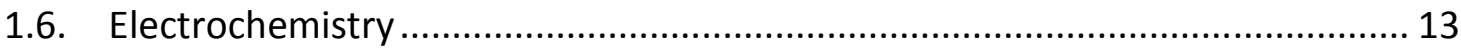

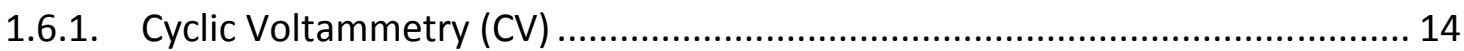

1.6.2. Relevant Electrochemical Reference Articles ............................................ 18

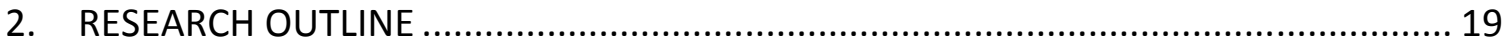

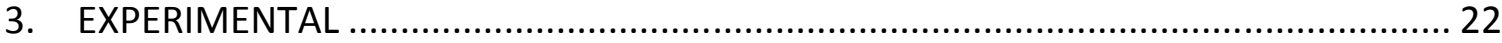

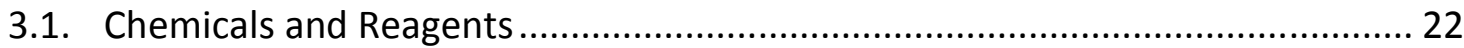

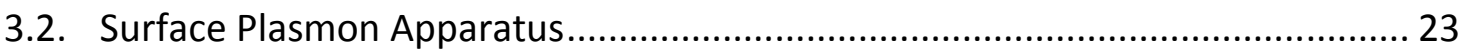

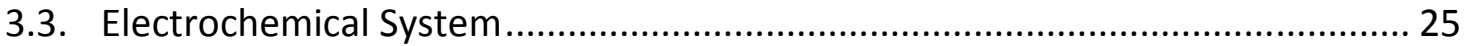

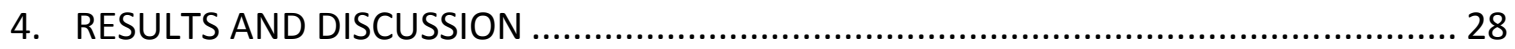

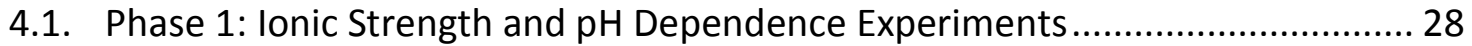

4.2. Rationale for Binary SAM and Potentiostatic Deposition Experiments .............. 34

4.3. Phase 2: Open Circuit Measurements of the $\mathrm{pH}$ Dependence of Binary SAM

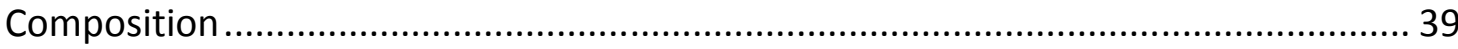

4.4. Phase 3: Potential Dependence of Monolayer Assembly .................................. 44

4.5. Phase 4: Closed Circuit Measurements of the $\mathrm{pH}$ Dependence of Binary SAM

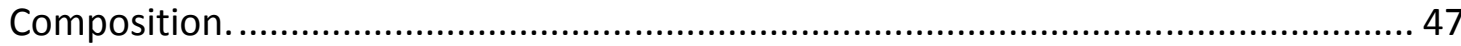

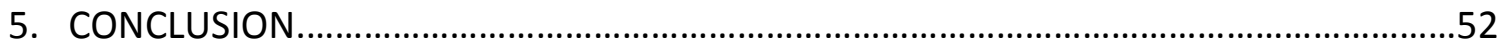

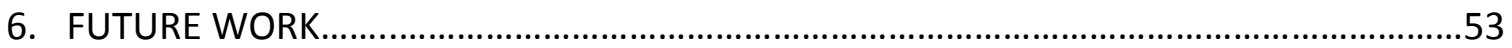

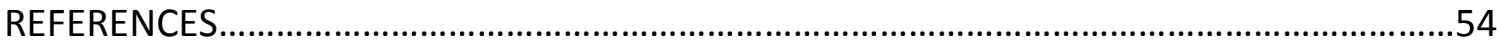




\section{LIST OF FIGURES}

Figure 1. Molecular structures of thiol compounds: (a) 3-mercapto-propionic acid (MPA); (b) 11-mercapto-undecanoic acid (MUA); and (c) 6-mercapto-1-hexanol $(\mathrm{MHX})$

Figure 2. Kretschmann configuration of surface plasmon resonance (SPR)..... 5

Figure 3. Schematic representation of a self-assembled alkanethiol molecule showing the tilt of the alkyl chain away from the perpendicular to the gold surface.

Figure 4. Typical cyclic voltammogram. Current $(A)$ is plotted vs. potential ( $m V$ vs.

$\mathrm{Ag} / \mathrm{AgCl}$ ) during a linear sweep of voltage from an initial point (left) and then the reverse from right to left.

Figure 5. Mechanism of ferrocene oxidation MUA monolayers on Au surface formed at $\mathrm{pH} 7$ (left) and $\mathrm{pH} 2$ (right) conditions.

Figure 6. Diagram of SPR reflectometer illustrating incident light beam and method of varying angle of incidence.

Figure 7. Illustration of cleaning and MUA assembly cycles used to repeat measurements on SPR sensor films.

Figure 8. Teflon ${ }^{\circledR}$ SPR electrochemistry cell and patterned Au SPR sensor on sapphire hemisphere surface.

Figure 9. Cartoon illustrating possible origin of decreased layer density in high $\mathrm{pH}$ and low ionic strength assembly conditions.

Figure 10. SPR sensograms reflecting chemisorption of 11-MUA anions ( $1.0 \mathrm{M}(\mathrm{A})$ to $0.00010 \mathrm{M}(\mathrm{E})$ in even decades) on gold surface from $\mathrm{pH} 7.2$ phosphate buffer $->$ buffer + 11-MUA (first arrow) -> pH 7.2 phosphate buffer (second arrow) as a function of time (sec) vs. $\Delta \lambda_{S P R}(\mathrm{~nm})$.......

Figure 11. SPR sensogram reflecting chemisorption of 11-MUA anions (1.0 $\mathbf{M}(A)$ to $0.00010 \mathrm{M}(\mathrm{E})$ in even decades) on gold surface from $\mathrm{pH} 7.2$ phosphate buffer $->$ buffer + 11-MUA (first arrow) -> pH 7.2 phosphate buffer (second arrow) as a function of time (sec) vs. $\Delta \lambda_{S P R}(\mathrm{~nm})$. 
Figure 12. Net SPR shift results from replicate adsorptions of MUA and MPA from low and high $\mathrm{pH}$ buffers prepared at a series of increasing ionic strengths. Error bars are 2-s standard deviations for triplicate runs done on the same day.

Figure 13. Cyclic voltammograms of $4 \mathrm{mM} \mathrm{Fe}(\mathrm{CN})_{6}^{-4}$ at $\mathrm{Au}$ (ex-situ, mechanically polished Au disk electrodes) coated with MUA (blue, $\mathrm{pH} 2$, and red, $\mathrm{pH} 7$ depositions) and MPA (green trace).

Figure 14. Open circuit SPR sensograms showing wavelength shift (nm) vs. time (sec) at $\mathrm{pH} 3,7$ and 11 for 11-MUA, 6-MHX, and mixed monolayer.

Figure 15. Open circuit bar graph showing delta-lambda-SPR (nm) at $\mathrm{pH} 3,7$, and 11 for 11-MUA, 6-MHX and mixed monolayer

Figure 16. Potentiostatic dependence experiment illustrating the potential dependence of the SPR wavelength as the potential is incremented from $-0.50 \mathrm{~V}$ to $+0.50 \mathrm{~V}$ before and after assembly of MUA 7D on gold surface.

Figure 17. Potential dependence plot of MUA, MUAMHX and MHX as a function of Potential (V vs. $\mathrm{Ag} / \mathrm{AgCl}$ ) vs. Corrected SPR Wavelength shift $(\mathrm{nm})$ on gold surface at $\mathrm{pH}$ 7.MUA/MHX and $\mathrm{MHX}$, a potential of $0.00 \mathrm{~V}$ was applied to the Au sensor surface.

Figure 18. Closed circuit SPR sensograms showing wavelength shift $(\mathrm{nm})$ vs. time (sec) at $\mathrm{pH} 3,7$, and 11 for $11-\mathrm{MUA}, 6-\mathrm{MHX}$, and mixed monolayer with $\mathrm{E}=0.00 \mathrm{~V}$..... 50

Figure 19. Closed circuit bar graph showing delta-lambda-SPR $(\mathrm{nm})$ at $\mathrm{pH} 3,7$ and 11 for 11-MUA, 6-MHX and mixed monolayer with $\mathrm{E}=0.00 \mathrm{~V}$ 


\section{LIST OF TABLES}

Table 1. Experimental parameters for thiol assembly experiments: MUA, MPA and MHX assembly on gold at $\mathrm{pH} 2,3,7$ and 11 and as a function of potential and buffer ionic strength. .... 


\section{INTRODUCTION}

This paper describes initial experimental efforts to fabricate nanoelectromechanically (NEM) active surface molecular layers. Hypothesized NEM layers will exhibit substantial structural rearrangement upon electrical stimulus and are important because they may enable active control of wetting, passivation or other interesting surface phenomena. Eventually, NEM active surfaces may selectively adsorb targets, selectively catalyze electrochemical reactions, or yield large electrowetting responses. At this stage, our primary interest is the formation of molecular layers on gold film surface plasmon sensor surfaces that are suitable anchor layers for subsequent tethering of NEM-active molecular components. We seek a better understanding of the factors controlling the composition of monolayers formed from mixtures of charged and neutral molecular components. The experiments described herein explore the $\mathrm{pH}$ and ionic strength effects that govern the self-assembly of such two-component systems. Using these insights, we wish to use these mixed layers as anchors to prepare covalently tethered molecular NEM surfaces. Our work thus far focuses on the limited goal of the controlled preparation of mixed monolayers of 6-mercaptohexanol (MHX), 11mercaptoundecanoic acid (MUA) and 3-mercaptopropionic acid (MPA) that may eventually serve as suitable anchor layers (through amide chemistry) for NEM layers. See Figure 1 for structures. The preparation of these mixed layers was monitored with surface plasmon resonance and cyclic voltammetry, and protocols for the reproducible generation of mixed layer composition were developed. 


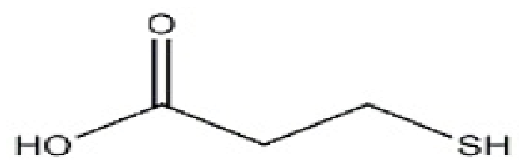

(a) MPA

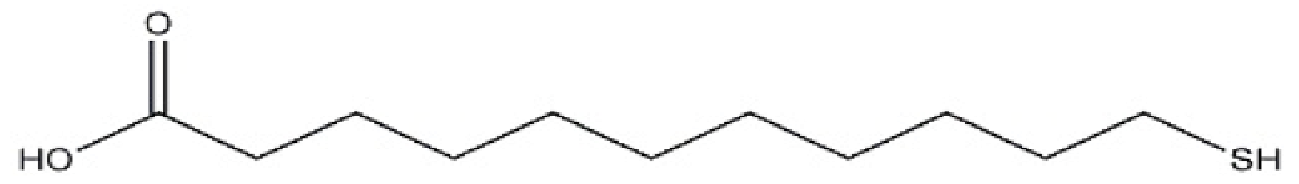

(b) MUA

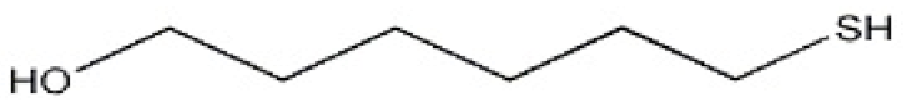

(c) $\mathrm{MHX}$

Figure 1. Molecular structures of thiol compounds: (a) 3-mercapto-propionic acid (MPA); (b) 11mercapto-undecanoic acid (MUA); and (c) 6-mercapto-1-hexanol (MHX) 


\subsection{Surface Plasmon Resonance (SPR)}

We use SPR to monitor the formation of the molecular layers used in this study. The following is a brief description of the essential aspects of SPR sensing. SPR, the way we implement it, is an optical technique that senses the refractive index of a medium near a thin ( $\sim 50 \mathrm{~nm}$ ) film of metal (typically gold) deposited on a glass substrate (see

Figure 2). SPR may be driven by p-polarized light as reflected at high angle from a glassgold film interface. This is referred to as the Kretschmann configuration and is a form of attenuated total internal reflection. In this setting, light is passed into a prism, one face of which is coated with a thin layer of Au. The light is then internally reflected by the metal film. The angle of incidence is set beyond the critical angle for total internal reflection, so the Au film would normally acts as a perfect mirror. But if a certain set of conditions is met, including specific values of refractive index for the prism, gold film, surface thickness and light wavelength, then the reflected light will pass through a sharp minimum at a particular angle. At this angle, surface plasmons are excited by the incident radiation, resulting in what is called surface plasmon resonance.

Surface plasmon waves are surface electromagnetic waves that propagate in the surface plane of a metal film and are a unique example of electrons interacting with

photons. This specific interaction induces a wave-like oscillation of the free electrons at the metal surface and thereby reduces the reflected light intensity. The SPR resonance angle (or wavelength) is the point at which a maximum loss of reflected light intensity is 
observed. This angle or wavelength is strongly dependent on the optical properties of the system such as the refractive index of the prism and of the medium contacting the Au film. The latter sensitivity accounts for how SPR reports interfacial binding, because the refractive index in the vicinity of the metal surface changes when molecules adsorb onto it. During a binding event, the temporal shift in $\lambda$-SPR or $\theta$-SPR in a flow-injection setting is characteristic of the binding interaction. Graphs of $\lambda$-SPR or $\theta$-SPR versus time are called sensograms. Sensograms record changes in SPR conditions as a function of time and thereby provide information on the kinetics and thermodynamics of the molecule adsorption onto the sensor surface. 


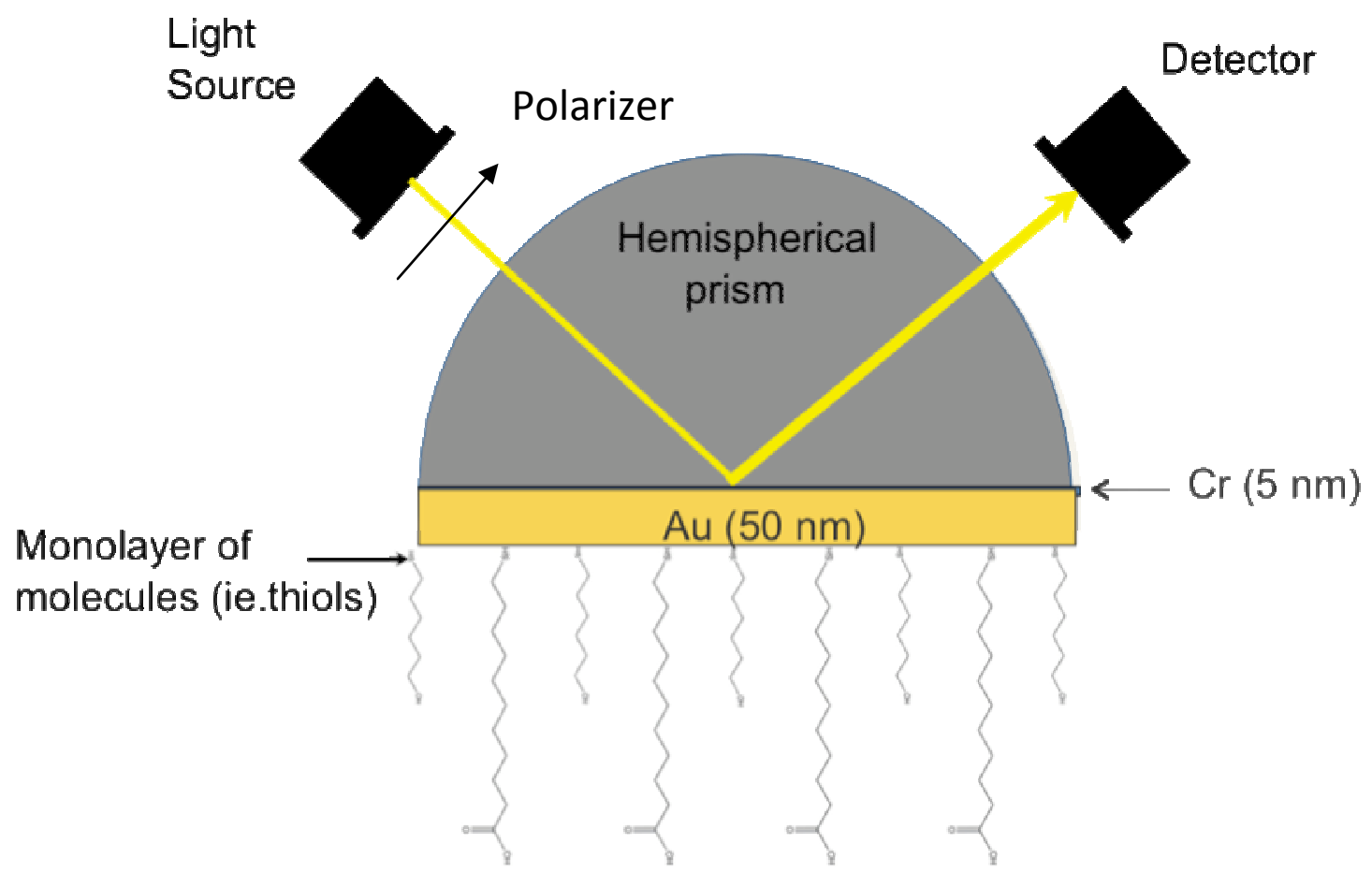

Figure 2. Kretschmann configuration of surface plasmon resonance (SPR). A light beam is incident onto a hemisphere, typically made of a high refractive index material such as sapphire. The apparatus is set up to either scan the incident angle (at constant light wavelength) or scan the wavelength at constant angle. For a critical combination of $\mathrm{Au}$ film thickness and monolayer side refractive index, the SP is excited and reflectivity is sharply attenuated. 


\subsection{Use of Surface Plasmon Resonance (SPR) as a Biosensor}

Molecular interactions that occur at the surface can be studied using instrumental techniques such as surface plasmon resonance (SPR), various fluorescence methods, scanning probe, e.g. scanning tunneling microscopy or atomic force microscopy, reflection absorption infrared spectroscopy (RAIRS), surface-enhanced Raman spectroscopy and electrochemical techniques such as cyclic voltammetry.

But SPR is an especially useful tool for studying biomolecular recognition at surfaces because it can monitor interactions, e.g., those between proteins and immobilized ligands in-situ, in real time and without fluorescent labeling. Labeling refers to the covalent modification of one or both of the interacting biomolecules with a fluorescent marker that later serves to report the interaction. A labeled analyte may be detected directly via its fluorescence or by a quenching or energy transfer, e.g., by Förster resonance energy transfer (FRET) interaction. But SPR detection requires no such label because it responds with extremely high sensitivity simply to surface refractive index. So, for example, interactions such as antibody-antigen, ligand-receptor and enzyme-substrate, as much as they involve the accumulation of relatively high index biomolecules at the sensor surface, may be detected via a shift in $\lambda$-SPR or $\boldsymbol{\theta}$-SPR. Because this shift occurs without covalent dye-modification of either of the interacting partners, SPR is relatively convenient. SPR biosensor devices exist today that have a detection limit of $\sim 1 \mathrm{pg} / \mathrm{mm}$ surface coverage allowing one to detect even low concentrations of low molecular weight analytes. ${ }^{1}$ 
The use of SPR in a biosensing context dates back to 1983, when Liedberg et al. ${ }^{2}$ first demonstrated the application of SPR-based sensors for monitoring of biomolecular interactions. But it was not until 1994 that the first substantial study of biospecific interactions appeared ${ }^{2}$. Currently, SPR is being utilized in biochemical research to study a broad range of biomolecular interactions including antigen-antibody, protein-protein or protein-DNA interactions and is also used to study conformational changes in surfacebound biomolecules ${ }^{2}$. This information is useful in fields such as medicine, biotechnology, pharmacology and food monitoring.

\subsection{Self Assembled Monolayer (SAM) Formation}

Self-assembly is a term used to describe processes in which a disordered system of pre-existing components forms an organized structure or pattern as a consequence of specific, local interactions among the components. Self-assembly of molecules at surfaces yields a self-assembled monolayer or "SAM".

Many systems can undergo surface self-assembly such as: long chain carboxylic acids $\left(\mathrm{C}_{n} \mathrm{H}_{2 n+1} \mathrm{COOH}\right)$ at metal oxide substrates, via chemisorption of $\mathrm{CO}_{2} \mathrm{H}$ to the metal oxide; organosilane species $\left(\mathrm{RSiX}_{3}, \mathrm{R}_{2} \mathrm{SiX}_{2}\right.$, or $\left.\mathrm{R}_{3} \mathrm{SiX}\right)$ where $\mathrm{R}$ is an alkyl chain and $\mathrm{X}$ is a chloro or alkoxy group via siloxane bond formation with surface hydroxyls on substrates such as glass, silicon and aluminum oxide; and organosulfur-based species at noble metal surfaces via metal-sulfur bond formation.

Monolayer films can also be prepared either using a Langmuir-Blodgett (LB) or a self-assembly technique. The LB method involves spreading an insoluble compound 
onto an aqueous phase and compressing the film mechanically until the molecules are densely packed and oriented normal to the surface. Once this occurs, the monolayer is transferred to a solid substrate via dipping ${ }^{3}$. The Langmuir-Blodgett (LB) method is useful when multilayers are preferred, but monolayers thus formed tend to undergo structural relaxation and are somewhat difficult to prepare.

Monolayer self-assembly relies on there being a strong interaction between the adsorbate, e.g., an alkanethiol, and the substrate, e.g., a gold surface, to form a monolayer film ${ }^{3}$. A widely used protocol for preparing alkanethiol SAMs on metal such as gold, silver, or palladium is to immerse the substrate into a dilute ethanolic solution ranging from 1 to $10 \mathrm{mM}$ of thiol for $\sim 12$ to 18 hours at room temperature. Typically, it only takes from seconds to minutes for the adsorbate to cover the substrate surface; however, it may require a much longer time for a dense and structurally organized SAM to form due to the relatively slow dynamics of surface molecular translation ${ }^{4}$. Disulfides (RSSR), thioethers (RSR) and thiols (RSH) can be used to form monolayers, but thiols are more frequently used due to their high solubility in solution. According to Whitesides et al. ${ }^{5}$, alkanethiol SAMs on gold are the best model system available to investigate the fundamental aspects of biointerfacial science. Since gold films support both the SPR effect and alkanethiol SAMs, they are a natural fit for our study.

Some details of the alkanethiol interfacial binding reaction may be instructive. For example, to the best of our knowledge upon binding to $\mathrm{Au}$, the hydrogen in the $\mathrm{S}-\mathrm{H}$ bond is lost perhaps as $\mathrm{H}_{2}$ or as water by reaction with traces of oxidants in the system 
as Bain et al. ${ }^{6}$ indicate. The Au-S binding mechanism likely involves an oxidative addition of the $\mathrm{S}-\mathrm{H}$ bond to the Au surface followed by a reductive elimination of the hydrogen atom.

Numerous studies have shown that monolayers formed from long-chain alkanethiols on gold are substantially ordered. The alkyl chains are mainly trans extended with the sulfur atoms resting in threefold hollows between Au atoms on the hexagonal Au (III) surface plane. Computer simulations have illustrated that the outermost end of the chains have greater mobility than the inner parts, and possibly have some gauche conformations in an otherwise allowed trans system ${ }^{3}$. But according to Bain et al. ${ }^{6}$ the water contact angle of n-alkanethiol SAMs alternates as an odd/even function of the number of carbons in the alkyl chain. C-terminal functionalized alkanthiols such as $\mathrm{HS}\left(\mathrm{CH}_{2}\right)_{n} \mathrm{NH}_{2}$ and $\mathrm{HS}\left(\mathrm{CH}_{2}\right)_{n} \mathrm{COOH}$ greatly decrease the water contact angle of the comprising SAM.

Kinetic studies of self-assembly of alkanethiols onto gold show that this process occurs in two distinguishable phases: an initial fast phase where the sulfur-containing compound is quickly chemisorbed to ca. $90 \%$ of its ultimate coverage onto the metal substrate and a second slow phase during which the asymptotic increases in alkanethiol occur concomitantly with alkyl chain rearrangement as the n-alkyl chains self-organize into an extended close packed, all trans conformation. As Figure $\mathbf{3}$ shows, the alkyl chains are tilted from the perpendicular to the gold surface with an angle, $\theta$, between $26^{\circ}$ and $28^{\circ}$. Distinct differences occur in the structure of the SAMs formed from long 
to short chain alkanethiols. Ellipsometric studies have shown that, as the chain length decreases, the degree of order of the SAMs decreases along with the packing density and surface coverage. ${ }^{7}$

\subsection{Mixed Monolayers}

Single-component thiol SAMs has been extensively studied ${ }^{7}$, but mixed, e.g. twocomponent or binary SAMs have received far less attention. Some examples of binary SAM studies include the following: Chen et al. ${ }^{7}$ illustrate that mixed SAMs offer the possibility to control the chemical and structural properties of a surface by adjusting the abundance, type and spatial distribution of the termini at the solution interface, for example to promote or inhibit protein adsorption due to the multiple chemical functionalities on the surface. Many examples of surface modification using twocomponent or "binary" SAMs exist including ones wherein composition is varied as a function of position. Love et al. ${ }^{4}$ used mixed SAMs for defining gradients of interfacial composition that, in turn, are useful for studying the adhesive properties and motive phenomena of adsorbed cells. 


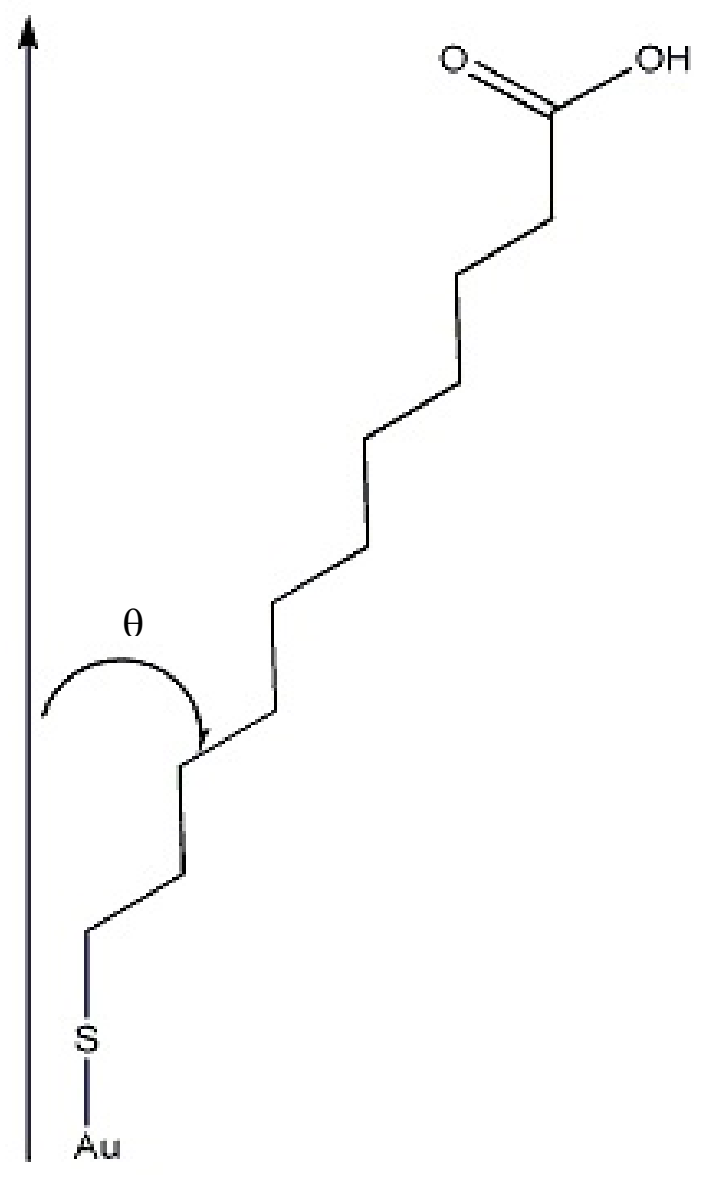

Figure 3. Schematic representation of a self-assembled alkanethiol molecule showing the tilt of the alkyl chain away from the perpendicular to the gold surface. 
One concern related to the preparation of binary SAMs by the simultaneous coadsorption from a two-component solution is the possibility of phase segregation of constituents in the SAM, particularly when molecules of different chain lengths are involved. So alternate methods for making binary SAMs have been explored including: adsorption of asymmetric disulfides (RSSR'), adsorption of asymmetric dialkylsulfides $\left(\mathrm{RSR}^{\prime}\right)$, insertion of second component into an incomplete SAM and chemical modification of terminal groups. In this research we use mixed thiol solutions, a simple approach, but one that is vulnerable to microscopic phase segregation. Unfortunately, we do not presently have the capability to evaluate this phenomenon, but attention to possible phase segregation remains a long-term objective.

\subsection{Research Objective}

The long-term goal of this research is to be able to create "open," low-density conformationally flexible monolayers that have an electromechanical response. This thesis is a first-step along this line and involves the study of how electrostatic repulsion between neighboring $\omega$-mercaptocarboxyate molecules tends to control the composition of single component and binary mixed SAMs. The tools we use in this work are surface plasmon resonance (SPR) and electrochemistry. Experiments are designed to answer the following question: How do experimental parameters such as solution $\mathrm{pH}$, ionic strength and electrochemical potential control the composition of $\omega$ - 
mercaptocarboxyate SAMs prepared from either the pure carboxylate or equimolar mixtures of the carboxylate and neutral molecules?

The simple hypothesis that guides the experiments herein is that the headgroup charge of $\omega$-mercaptocarboxylic acids will limit their adsorption density in a $\mathrm{pH}$ and ionic strength dependent fashion. High $\mathrm{pH}$ and low ionic strength are expected to contribute to low surface density of the carboxylate components. The utility of the study is ultimately to find the conditions under which mixed SAMs thus formed may serve as suitable anchor layers for a secondary, NEM active layer such as a flexible oligomer that is terminated in charged chromophore.

\subsection{Electrochemistry}

Electrochemistry is the study of chemical reactions that are accompanied by the transfer of electrons and charge when a reaction occurs. When a voltage is applied to an electrode surface, electrons may be transferred to or from an electroactive species in solution such as ferrocene ferricinium: $\mathrm{FeCp}_{2} \leftrightarrow \mathrm{FeCp}_{2}^{+}+\mathrm{e}^{-}$. An oxidation reaction occurs when electrons are transferred to the electrode from the electroactive species, and, a reduction reaction occurs when electrons are transferred from the electrode to the electroactive species.

Typically, a three-electrode cell is used which is comprised of a working electrode, a reference electrode and a counter or auxiliary electrode. In this setting, the potential of the working electrode is controlled relative to the reference electrode using the counter electrode to supply the necessary current. A common aqueous reference 
the electrode is silver chloride electrode $(\mathrm{Ag} / \mathrm{AgCl})$. The auxiliary electrode is used to supply the current for the electrochemical reaction, and is typically either platinum or gold, since these noble metals do not appreciably dissolve.

\subsubsection{Cyclic Voltammetry (CV)}

Cyclic voltammetry is a popular electrochemical technique for studies of molecular order and surface coverage of SAMs. In CV, a linear voltage ramp is applied to the electrode and the current response is measured. The voltage program applied to the working electrode is specified by setting the initial potential $\left(V_{1}\right)$ and a vertex potential $\left(V_{2}\right)$. As Figure 4 shows, when $V_{1}$ is applied to the working electrode, the potential increases linearly until it reaches $V_{2}$ and then it scans in back to $V_{1}$. The result is a cyclic voltammogram plot of current (I) vs. potential (E). The cyclic voltammogram normally exhibits peaks, identified by both their potential (e.g. E $E_{P C} E_{P A}$ for peak cathodic or anodic potentials) and their currents (e.g. i PC for peak cathodic current, etc.) as the potential are swept past the redox potential $\left(E^{o^{\prime}} \approx\left(E_{P C}+E_{P A}\right) / 2\right)$ of the electroactive species. These peak currents are proportional to the flux of electroactive species to the electrode surface to the rate of electron transfer between the species and the electrode. The flux of redox species is in turn proportional to the concentration and diffusion coefficient of the redox species. But current is ultimately limited by the molecular scale access scale that the redox species has to the electrode surface and to the intrinsic kinetics of the electron transfer reaction. Alkanethiol monolayers can substantially attenuate the currents seen in a CV by reducing access of the electroactive 
species to the surface and by reducing electron transfer rates, so CV may be used as a diagnostic of SAM integrity. This is illustrated in Figure 5, which depicts electron transfers at relatively permeable (left) and dense (right) MUA layers. On the right hand side, it is clear that electron transfers must take place over a long distance because the alkyl chain is a good dielectric - this dramatically attenuates the electron transfer rates. 


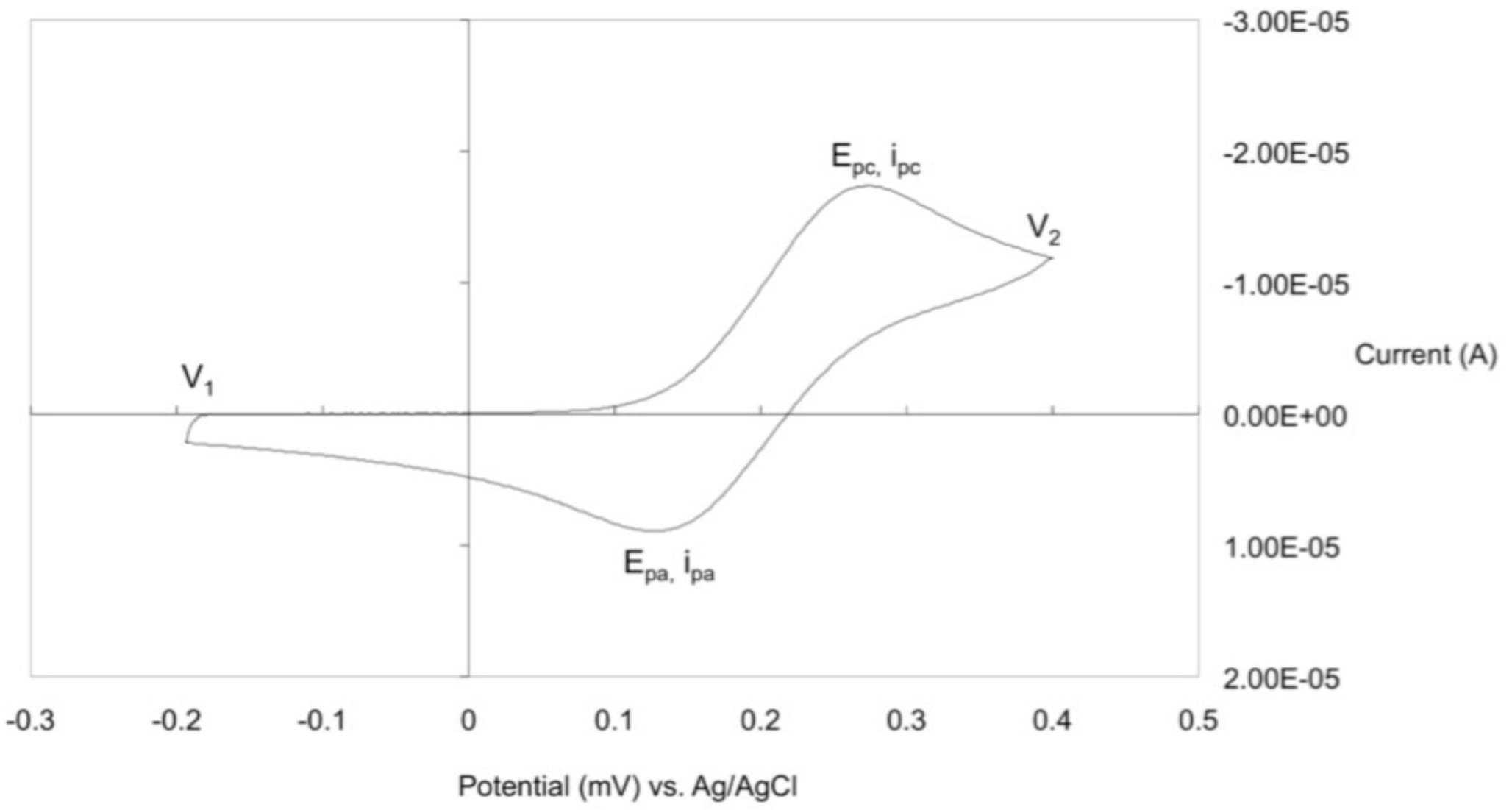

Figure 4. Typical cyclic voltammogram. Current $(A)$ is plotted vs. potential ( $\mathrm{mV} v \mathrm{~s} . \mathrm{Ag} / \mathrm{AgCl}$ ) during a linear sweep of voltage from an initial point (left) and then the reverse from right to left. Note $E_{p c}$ is the cathodic peak potential and $E_{p a}$ is the anodic peak potential. 

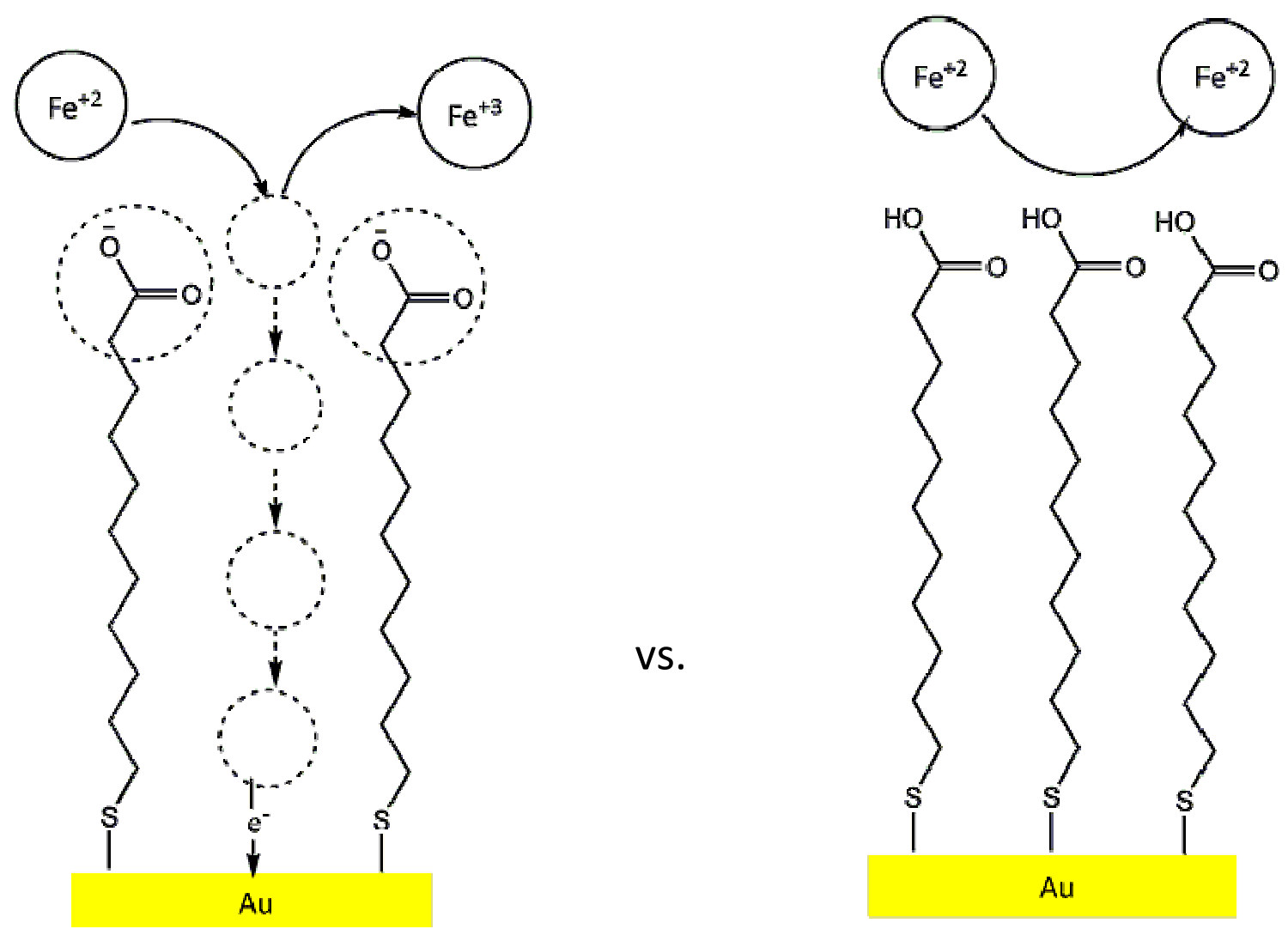

Figure 5. Mechanism of ferrocene oxidation MUA monolayers on Au surface formed at $\mathrm{pH} 7$ (left) and pH 2 (right) conditions. 


\subsubsection{Relevant Electrochemical Reference Articles}

The dependence of the monolayer integrity on the substrate potential was investigated using grazing incidence reflection-absorption FTIR, CV and electrochemical impedance spectroscopy (EIS) by Diao et al. ${ }^{8}$. Cyclic voltammetry results show that substrate potential did, in fact, have a substantial effect on the integrity of the alkanethiol SAMs, where the best film integrity on gold was found to be at $\sim 0.4 \mathrm{~V}$ (vs. $S C E)$.

Similarly, Arakawa et al. ${ }^{9}$ examined the effect of the charged state of a gold substrate on the surface composition of a phase-separated binary SAM, 1tetradecanethiol (TDT) and 3-mercapto-1-propanol (MPOH), using cyclic voltammetry of the reductive desorption of SAMs. As it was found that by keeping the gold substrate potential to a less negative value of $\sim-0.500 \mathrm{~V}$ compared to that of $-0.800 \mathrm{~V}$ in the bathing ethanolic solution, the adsorptivity of $\mathrm{MPOH}$ was enhanced on the substrate through a reduction reaction.

Alkanethiol SAMs can also be removed electrochemically. Applying sufficient cathodic potential to a gold electrode modified with a thiolated SAM in alkaline electrolyte can cause a one electron reductive desorption of the thiol:

$\mathrm{RS}-\mathrm{Au}+1 \mathrm{e}^{-} \rightarrow \mathrm{RS}^{-}+\mathrm{Au}$, which is a process referred to as cathodic desorption by Widrig et al. ${ }^{10}$. Widrig studied the process over a range of $\mathrm{pH}$ conditions and using ellipsometry and infra-red spectroscopy. She concluded that, upon adsorption at both $\mathrm{Au}$ and $\mathrm{Ag}$ surfaces, the sulfur atom is oxidized by one electron ${ }^{10}$. Similarly, Calvente ${ }^{11}$ 
and Loglio ${ }^{12}$ have studied oxidative desorption processes of alkanethiolate SAMs. They studied the oxidative desorption of 2-mercaptoethanesulfonate from Au electrodes in $\mathrm{NaOH}$ and found that the process involved a total of around 12 electrons. The consensus from these studies is that number of electrons being transferred in the oxidative desorption of the thiol from the gold surface depends on the particular reaction conditions since the initial oxidative cleavage of the gold-sulfur bond is followed by further oxidation of the sulfur. In this thesis, electrochemical potential control is used both to control deposition and to remove SAMs from the Au surface when that is needed.

\section{RESEARCH OUTLINE}

In this research, SAMs of MUA, MPA, MHX and mixtures of MHX and MUA were assembled under a variety of $\mathrm{pH}(2,3,7$ or 11$)$, ionic strength $\left(\mu \approx 10^{-4}, 10^{-3} \ldots 10^{0} \mathrm{M}\right)$ and electrochemical potentials (open circuit, $-0.50,-0.25,0.00,0.25,0.50 \mathrm{~V} \mathrm{vs.} \mathrm{Ag} / \mathrm{AgCl}$ ) to examine the possible influence of these variables on the amount of thiol that is immobilized. As noted earlier, the overriding question we seek to answer is how these assembly conditions influence the composition of the resulting SAMs, but we also have sought to establish a clear picture of the reproducibility of our assembly results. Monolayer assembly was mentioned using both SPR and electrochemical techniques. The SPR measurement was made continuously before, during and after thiol layer assembly, but the electrochemical measurement was made periodically, i.e., before and 
after the alkanethiol SAMs are formed. We also employed cyclic voltammetry to measure the permeability of the SAM using ferrocene as a 'probe' redox molecule.

Four phases of experiments were implemented as summarized in Table 1. In the first phase, open-circuit (i.e. no electrochemical control) monolayer assembly experiments were performed as a function of the $\mathrm{pH}$ and approximate ionic strength of the buffer solutions and the results that compared to single-component thiol solutions of MPA, MUA and MHX (as a control). In the second phase, equimolar mixed $10^{-4} \mathrm{M}$ MUA-MHX solutions were compared to pure MUA and MHX, and across $\mathrm{pH} 3,7$ and 11 but setting the ionic strength at $10^{-3} \mathrm{M}$ throughout. The third phase was an initial study into the potential dependence of the assembly process conducted at $\mathrm{pH} 7$ but across a range of constant potentials values. In this phase, $\mathrm{pH} 7$ solutions of thiol as in phase 2 were assembled onto $\mathrm{Au}$ at $-0.50,-0.25,0.00,0.25$ and $0.50 \mathrm{~V} \mathrm{vs.} \mathrm{Ag} / \mathrm{AgCl}$. Lastly, in phase 4 , the experiments in phase 2 were reproduced but at a controlled potential of $0.00 \mathrm{~V}$ vs. $\mathrm{Ag} / \mathrm{AgCl}$. 
Table 1. Experimental parameters for thiol assembly experiments: MUA, MPA and MHX assembly on gold at $\mathrm{pH} 2,3,7$ and 11 and as a function of potential and buffer ionic strength. Checked symbols $(\sqrt{ })$ represent triplicate assembly runs performed at those particular concentrations of phosphate buffer. Dashes (-) indicate runs omitted.

$$
\log [\mu / M]
$$

Assembly Conditions

\begin{tabular}{|c|c|c|c|c|c|c|c|}
\hline & & & 0 & -1 & -2 & -3 & -4 \\
\hline \multirow{6}{*}{$\begin{array}{l}\text { Phase 1: Open } \\
\text { Circuit, Single } \\
\text { Component, } \\
\text { lonic Strength } \\
\text { and pH } \\
\text { Dependence }\end{array}$} & \multirow{3}{*}{$\begin{array}{c}\text { Neutrals } \\
\text { at } \mathbf{p H} \mathbf{2}\end{array}$} & MUA & $\sqrt{ }$ & $\sqrt{ }$ & $\sqrt{ }$ & - & - \\
\hline & & MPA & $\sqrt{ }$ & $\sqrt{ }$ & $\sqrt{ }$ & - & - \\
\hline & & $\mathrm{MHX}$ & - & - & - & - & - \\
\hline & \multirow{3}{*}{$\begin{array}{l}\text { Anions } \\
\text { at pH } 7\end{array}$} & MUA & $\sqrt{ }$ & $\sqrt{ }$ & $\sqrt{ }$ & $\sqrt{ }$ & $\sqrt{ }$ \\
\hline & & MPA & $\sqrt{ }$ & $\sqrt{ }$ & $\sqrt{ }$ & $\sqrt{ }$ & $\sqrt{ }$ \\
\hline & & $\mathrm{MHX}$ & $\sqrt{ }$ & $\sqrt{ }$ & $\sqrt{ }$ & $\sqrt{ }$ & $\sqrt{ }$ \\
\hline \multirow{9}{*}{$\begin{array}{l}\text { Phase 2: Open } \\
\text { Circuit, Mixed } \\
\text { Layer, pH } \\
\text { Dependence } \\
\text { Study }\end{array}$} & \multirow{3}{*}{ pH 3} & $\mathrm{MHX}$ & - & - & - & $\sqrt{ }$ & - \\
\hline & & MUA-MHX & - & - & - & $\sqrt{ }$ & - \\
\hline & & MUA & - & - & - & $\sqrt{ }$ & - \\
\hline & \multirow{3}{*}{ pH 7} & $\mathrm{MHX}$ & - & - & - & $\sqrt{ }$ & - \\
\hline & & MUA-MHX & - & - & - & $\sqrt{ }$ & - \\
\hline & & MUA & - & - & - & $\sqrt{ }$ & - \\
\hline & \multirow{3}{*}{ pH 11} & $\mathrm{MHX}$ & - & - & - & $\sqrt{ }$ & - \\
\hline & & MUA-MHX & - & - & - & $\sqrt{ }$ & - \\
\hline & & MUA & - & - & - & $\sqrt{ }$ & - \\
\hline \multirow{5}{*}{$\begin{array}{l}\text { Phase 3, Closed } \\
\text { Circuit, Mixed, } \\
\text { pH } 7 \\
\text { Electrochemical } \\
\text { Potential }\end{array}$} & \multicolumn{2}{|l|}{$-0.50 \mathrm{~V}$} & - & - & - & $\sqrt{ }$ & - \\
\hline & $-0.25 \mathrm{~V}$ & MHX & - & - & - & $\sqrt{ }$ & - \\
\hline & $0.00 \mathrm{~V}$ & MUA-MHX & - & - & - & $\sqrt{ }$ & - \\
\hline & $+0.25 \mathrm{~V}$ & MUA & - & - & - & $\sqrt{ }$ & - \\
\hline & $+0.50 \mathrm{~V}$ & & - & - & - & $\sqrt{ }$ & - \\
\hline \multirow{9}{*}{$\begin{array}{l}\text { Phase 4: Closed } \\
\text { Circuit, } 0.00 \text { V } \\
\text { vs. Ag/AgCl, } \\
\text { Mixed Layer, } \\
\text { pH Dependence } \\
\text { Study }\end{array}$} & \multirow{3}{*}{ pH 3} & $\mathrm{MHX}$ & - & - & - & $\sqrt{ }$ & - \\
\hline & & MUA-MHX & - & - & - & $\sqrt{ }$ & - \\
\hline & & MUA & - & - & - & $\sqrt{ }$ & - \\
\hline & \multirow{3}{*}{ pH 7} & $\mathrm{MHX}$ & - & - & - & $\sqrt{ }$ & - \\
\hline & & MUA-MHX & - & - & - & $\sqrt{ }$ & - \\
\hline & & MUA & - & - & - & $\sqrt{ }$ & - \\
\hline & \multirow{3}{*}{ pH 11} & $\mathrm{MHX}$ & - & - & - & $\sqrt{ }$ & - \\
\hline & & MUA-MHX & - & - & - & $\sqrt{ }$ & - \\
\hline & & MUA & - & - & - & $\sqrt{ }$ & - \\
\hline
\end{tabular}




\section{EXPERIMENTAL}

\subsection{Chemicals and Reagents}

11-mercapto-undecanoic acid (MUA) and 6-mercapto-1-hexanol (MHX) were obtained from Aldrich Chemical Corporation and Fluka Corporation, respectively. Acetonitrile (Sigma-Aldrich), Rhodamine 6G (Matheson Coleman \& Bell), potassium ferrocyanide (J.T. Baker Chemical Co.) and ferrocene (sublimed, Aldrich) were provided by San Jose State University. Lithium perchlorate (Aldrich), gold and chromium (AlfaAesar) for the preparation of SPR sensor films were of high purity ( $\geq 99.99 \%)$. Potassium phosphate buffer solutions were prepared from 1.00 M phosphoric acid (Sigma Ultra) and then titrated to desired $\mathrm{pH}$ with $50 \% \mathrm{KOH}$ solutions. Subsequent solutions were prepared by 10 -fold serial dilutions of the $1 \mathrm{M}$ buffers to a final concentration of $10^{-4} \mathrm{M}$ and $\mathrm{pH}$ adjusted with microliter aliquots of $50 \% \mathrm{KOH}$ or $35 \% \mathrm{H}_{3} \mathrm{PO}_{4}$ solutions. Because MUA is poorly soluble in water, blank and sample solutions for SPR analysis were mixed with $10 \%$ ethanol (Fisher Scientific). SPR quality solutions were prepared by mixing $45.000 \pm 0.005 \mathrm{~g}$ aliquots of the above aqueous phosphate buffers with exactly $5.000 \pm$ $0.005 \mathrm{~g}$ of denatured ethanol to yield solutions that were precisely $10.00 \%$ ethanol by weight. In this way the refractive indexes of the blank and thiol buffer solutions were nearly perfectly matched. In the case of thiol containing solutions, the ethanol was prepared to contain $1.0 \mathrm{mM}$ alkanethiol, yielding $0.10 \mathrm{mM}$ thiol in solutions for SPR experiments. Experiments with $1 \mathrm{mM}$ MHX or $1 \mathrm{mM}$ MUA in ethanol were periodically performed as control calibrants. Gold sensor films $(50 \mathrm{~nm})$ and $\mathrm{Cr}$ adhesion layers (5 
$\mathrm{nm}$ ) were thermally evaporated onto a polished sapphire hemisphere at ca. $5 \times 10^{-6}$ Torr and at $0.4 \mathrm{~nm} / \mathrm{s}$.

\subsection{Surface Plasmon Apparatus}

An incandescent visible light source was coupled via an optical fiber, focusing lens and an elliptical mirror into a $25 \mathrm{~mm}$ diameter sapphire hemisphere directly coated with $\operatorname{Cr}(50 \AA)) \mid A u(500 \AA ̊)$. Reflected light was collected into a second fiber and was analyzed with an Ocean Optics S2000 spectrometer. Solutions were transported with $\mathrm{N}_{2}$ pressure, (5-10 PSI) applied to the headspace of the solutions. A computer-controlled, zero dead volume selection valve (Upchurch Scientific) determined the timing of solvent and gas delivery to the cell. This system is illustrated in Figure 6. 


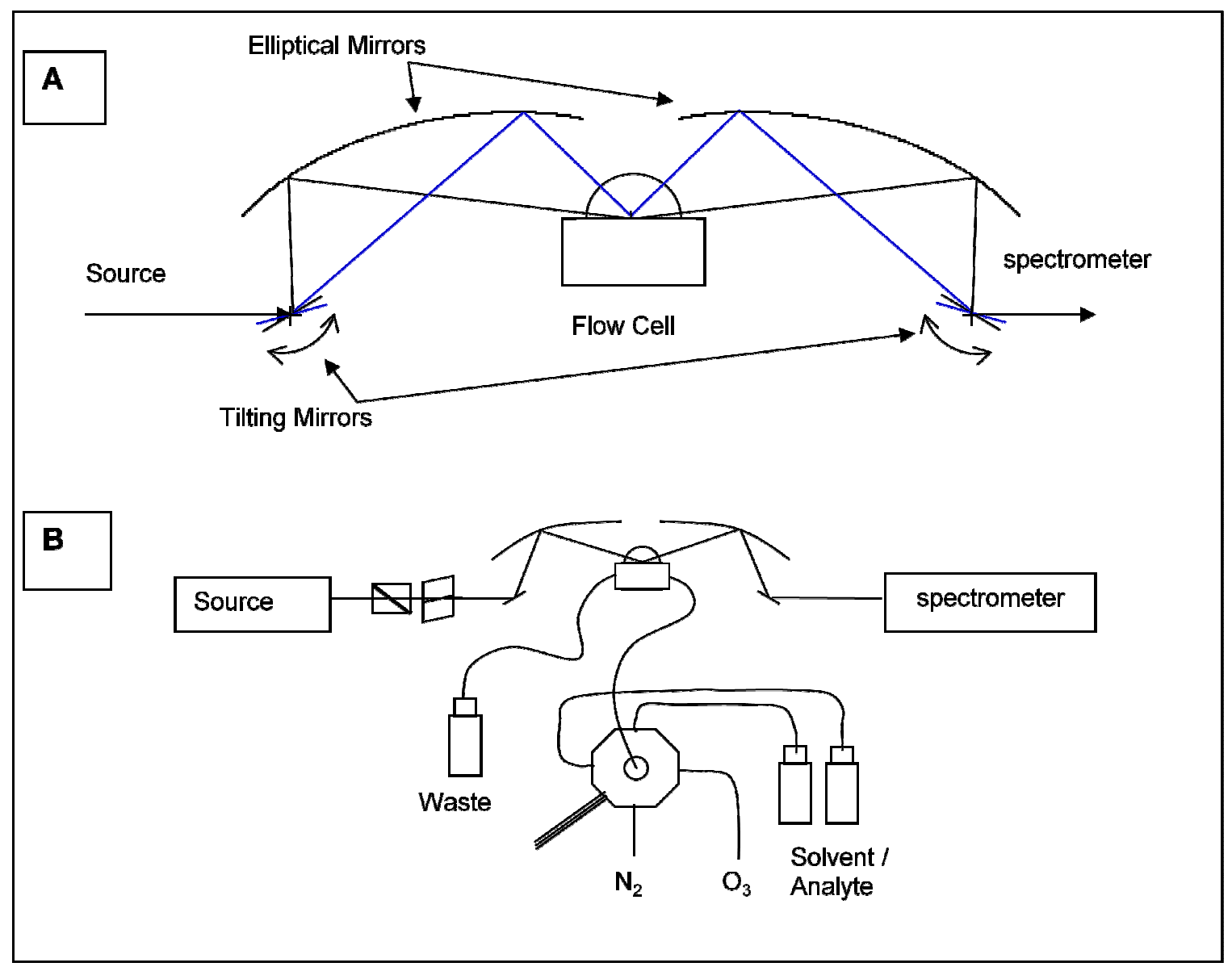

Figure 6. Diagram of SPR reflectometer illustrating incident light beam and method of varying angle of incidence. The broadband light is incident on the sapphire hemisphere and reflects off the Au sensor film that is in contact with liquid via the flow cell.

Collected light is analyzed by a CCD spectrometer. Note that for later studies the source and spectrometer were placed at a fixed angle of incidence ( $60^{\circ}$ from normal incidence). Nitrogen, ozone and various solutions are directed to the cell via a computer controlled selection valve under $\mathrm{N}_{2}$ pressure and then to waste. 


\subsection{Electrochemical System}

Electrochemical experiments were always done with three electrodes, a working electrode $(\mathrm{Au})$, a reference electrode $(\mathrm{Ag} / \mathrm{AgCl})$ and auxiliary electrode (Pt or $\mathrm{Au})$. Experiments were performed on an EG\&G Princeton Applied Research (PAR) Potentiostat / Galvanostat Model 263 and PAR Model 270 Electrochemistry software. Some experiments were performed ex-situ on a $2 \mathrm{~mm}$ diameter Au disc working electrode (Bioanalytical Systems, Inc.) that was prepared for experiments by mechanical polishing with $\mathrm{Al}_{2} \mathrm{O}_{3}$ polishing compounds (Buehler) followed by rinsing, sonicating in pure water and ethanol and, finally, drying with nitrogen. MUA and MPA depositions onto this electrode were carried out in quiescent solutions for a total of 5 minutes from buffers identical to those used in SPR experiments (Figure 7). Other experiments were performed in-situ on the Au SPR sensor films, in which case Au surface cleaning and pretreatment was done by electrochemical cycling between -1.4 and $+0.8 \mathrm{~V}$ vs. $\mathrm{Ag} / \mathrm{AgCl}$ in flowing $\mathrm{pH} 11$ phosphate buffer solutions. Electrochemical probe experiments were performed immediately preceding and immediately following SAM depositions in $0.10 \mathrm{M} \mathrm{LiClO}_{4}$ in acetonitrile electrolyte containing $1.0 \mathrm{mM}$ ferrocene using the configuration detailed in Figure 8. 


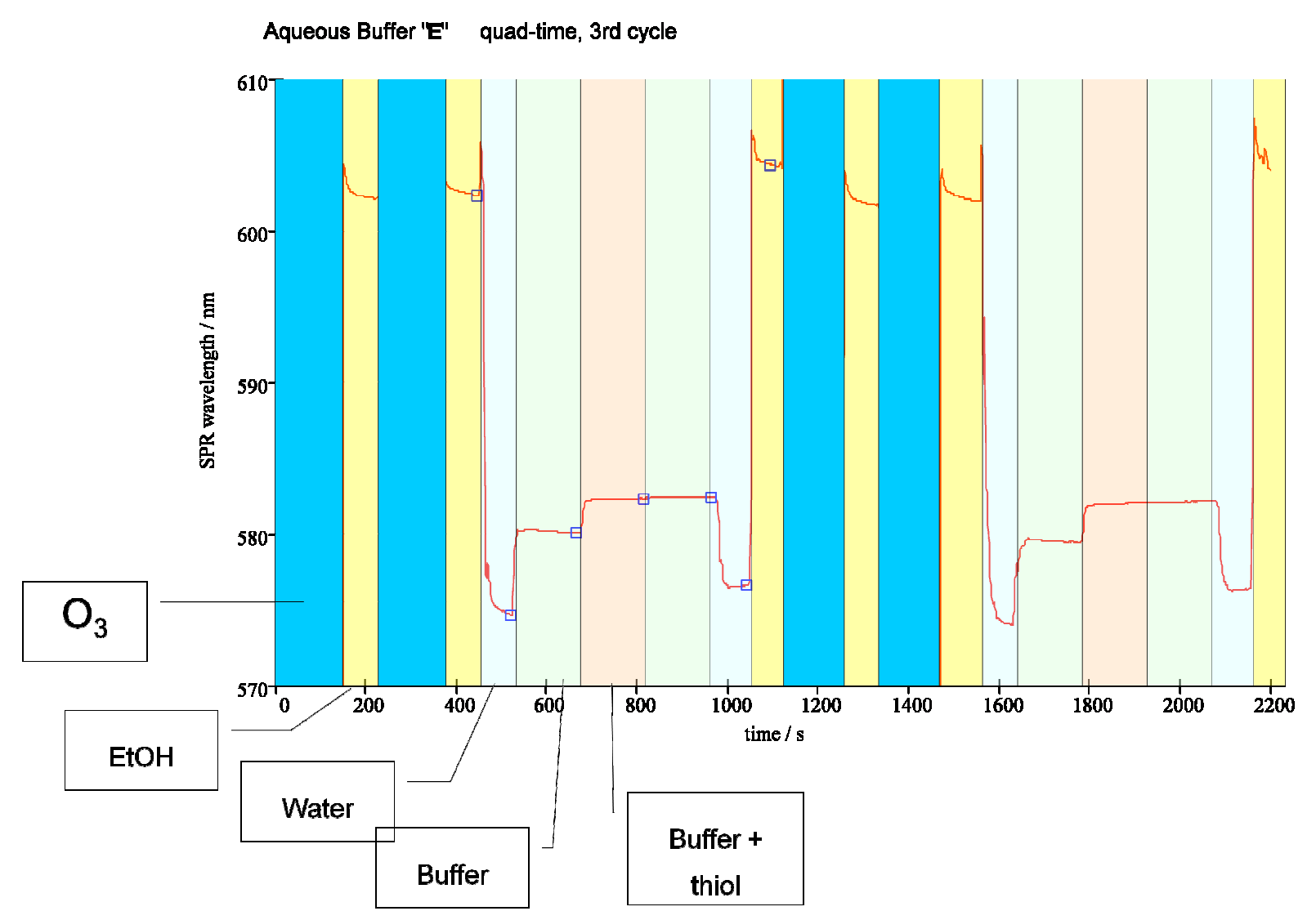

Figure 7. Illustration of cleaning and MUA assembly cycles used to repeat measurements on SPR sensor films. The red line denotes the SPR wavelength as the flow cell is filled with a series of different solutions. Note that during ozone $\left(\mathrm{O}_{3}\right)$ exposure intervals (which include bracketing $\mathrm{N}_{2}$ flows to dry the cell before ozone introduction) the surface plasmon is not resonant due to the low refractive index of the gas relative to solution; hence these time intervals are blocked out. Note the small but reproducible assembly transients observable just following 600 and $1600 \mathrm{~s}$. 


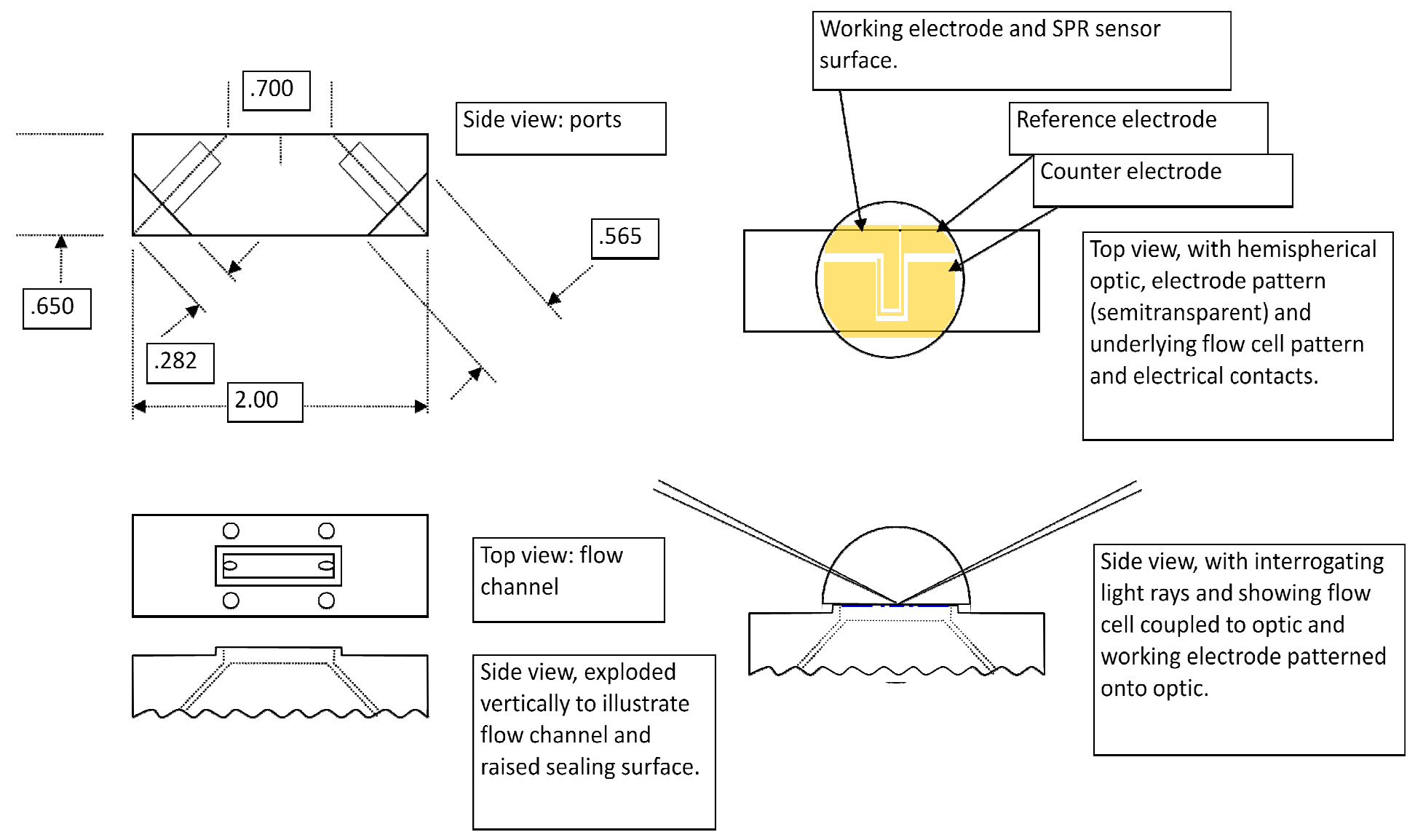

Figure 8. Teflon ${ }^{\circledR}$ SPR electrochemistry cell and patterned Au SPR sensor on sapphire hemisphere surface: Top left and bottom left figures illustrate the dimensions of Teflon flow cell ports, flow channel and the raised sealed surface from top and side views, respectively. Bottom right figure shows a side view of hemisphere with the transferred pattern placed on top of Teflon flow cell with interrogating light rays. 
The Au film SPR sensor and working electrode was patterned onto the cell using a simple laser-printer toner lift-off method. Briefly, the underlying Au electrode pattern (top right Figure 8 above) was printed onto a transparency and then transferred onto the flat surface of the hemisphere using a household iron. Chromium and gold were then deposited over the toner pattern. The Au-coated hemisphere was then sonicated for five minutes in acetone, which removes toner and lifts off the Au. The resulting patterned Au SPR electrochemistry sensor is then coupled to the Teflon ${ }^{\circledR}$ flow cell and sealed with static pressure against the raised ( 0.002 ") lip of the $3 / 16^{\prime \prime}$ wide $\times 0.7$ " long by 0.1 " deep flow channel. This cell design means that solutions contact only Teflon, gold, silver, platinum, sapphire and PEEK fittings, and no elastomers or other materials that might absorb thiol components or react deleteriously with ozone.

\section{RESULTS AND DISCUSSION}

\subsection{Phase 1: Ionic Strength and pH Dependence Experiments}

The first experimental phase involved experiments where solution ionic strength and $\mathrm{pH}$ effects on the chemisorption of MUA, MPA, and MHX were studied following the parameters shown in Phase 1 of Table 1. We expected to form open or relatively low density MUA monolayers when layers were adsorbed from high $\mathrm{pH}(\mathrm{pH}=7)(>>$ carboxylate $\mathrm{pK}_{\mathrm{a}} \sim 4$ ) and low ionic strength conditions were employed since under these conditions the carboxylate head groups should be predominantly ionized (at least in solution) and relatively poorly screened from one-another (Figure 9). Thus, a series of adsorption experiments were carried out as a function of $\mathrm{pH}$ and ionic strength. 


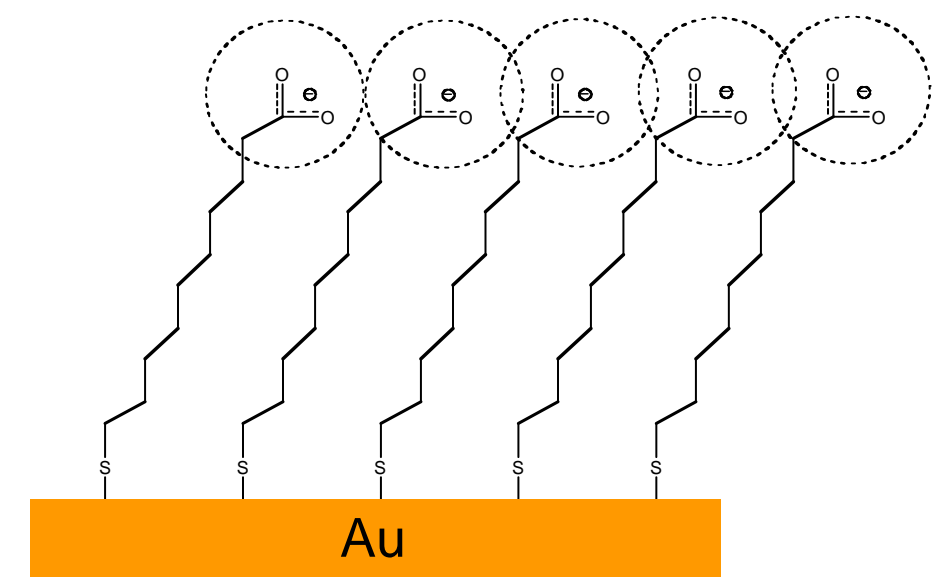

\section{pH 2.0}

High ionic strength -->

Denser layers

Larger SPR shifts

Lower voltammetric permeability

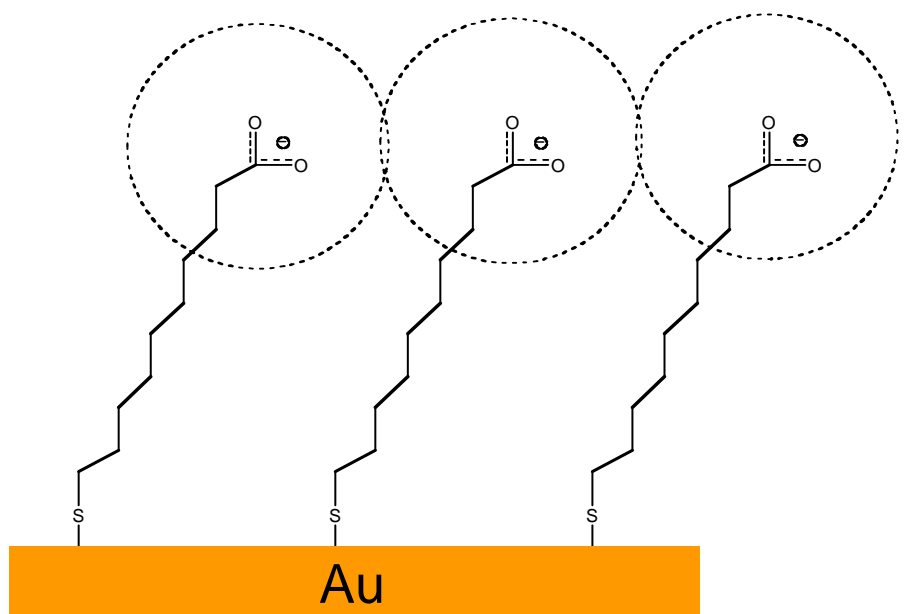

\section{$p H 7.0$}

Low ionic strength -->

Open layers

Figure 9. Cartoon illustrating possible origin of decreased layer density in high $\mathrm{pH}$ and low ionic strength assembly conditions. 
SPR sensogram plots of SPR wavelength shift, $\Delta \lambda_{\mathrm{SPR}}(\mathrm{nm})$ vs. time (sec) in Figure 10 illustrate results for the chemisorption of a series of MUA layers onto an Au surface and utilizing a series of solutions of increasing ionic strength. The flow-programs include a water baseline followed by blank buffer baseline and then a transition to thiolcontaining buffer, a blank buffer rinse and then a return to water. In-between water baselines, duplicate cleaning sequences comprising rinses with ethanol, $\mathrm{N}_{2}$ (to dry the surface), ozone, $\mathrm{N}_{2}$, and ethanol are performed in order to restore a clean Au sensor surface. An illustration of this cleaning cycle is given in Figure 7. In Figure 10, MUA adsorption data are plotted without adjustment along their wavelength dimensions. The water baselines are relatively well reproduced, but note that the high ionic strength of the 0.1 and $1 \mathrm{M}$ buffers elevates these buffer baselines above the others. The thiol adsorption transients are clearly comparable qualitatively, and none exhibit significant non-specific shifts when the solution flow is restored to the blank buffer indicating that there is little difference in bulk refractive index between the blank and $0.1 \mathrm{mM}$ thiol solutions. Hence, the entirety of the vertical displacement evident at $\sim 3600 \mathrm{~s}$ may be attributed to the accumulation of MUA at the sensor surface. The accumulation may also be noted in the change in SPR wavelength between the clean Au water rinse at the left and the MUA coated Au water rinses at the right. In Figure $\mathbf{1 1}$ the same data are plotted but after subtraction of the SPR wavelength recorded just prior to the introduction of thiol - i.e. with all buffer baselines normalized to zero. The results clearly show that the net $\Delta \lambda_{S P R}$ shift for MUA remained invariant to solution ionic 
strength across a range of buffer ionic strengths from $0.0001 \mathrm{M}$ (connoted buffer ' $\mathrm{E}^{\prime}$ ) to $0.1 \mathrm{M}\left({ }^{\prime} \mathrm{B}^{\prime}\right)$, but, abruptly and corresponding to the transition from $0.1 \mathrm{M}(\mathrm{B})$ and $1 \mathrm{M}(\mathrm{A})$ phosphate buffer ionic strength, there was a large and reproducible increase in the net $\Delta \lambda_{\text {SPR }}$ for the adsorption, the latter increase being precisely reproducible. The question arises therefore as to how to understand this unusually large $\Delta \lambda_{\text {SPR }}$ attributable exclusively to the $1 \mathrm{M}$ buffer. The water baselines evident in Figure $\mathbf{1 0}$ before and after the adsorption do not appear to differ substantially between low and high ionic strengths. But these baselines, alas, exhibit a much more substantial scatter and this may be obscuring a real difference. On the other hand, it is possible that the larger shift observed for the $1 \mathbf{M}$ case may be due to a difference in the SPR sensitivity at the slightly longer SPR wavelengths corresponding to the higher index $1 \mathbf{M}$ buffer. A block of experimental $\Delta \lambda_{\text {SPR }}$ shift results was acquired to help clarify this. These experiments were performed homologous to those illustrated in Figure 12. The block of experiments included the short-chain $\omega$-mercaptocarboxylate MPA, the neutral MHX as a control and experiments at pH 2 as indicated in Table 1, Phase 1. The average net shifts as a function of ionic strength from triplicate runs under the specified conditions are illustrated in Figure 12. The only species that experienced a substantial sensitivity to ionic strength were MUA and MPA at pH 7. The neutrals, MUA and MPA at pH 2 and $\mathrm{MHX}$ at $\mathrm{pH} 7$ were all invariant to ionic strength. Also noteworthy is the observation that at $\mathrm{pH} 2$, the $\Delta \lambda_{\text {SPR }}$ values for MUA and MPA were substantially larger than those observed at pH 7. In aggregate, the above results are all consistent with a denser layer 
formation at $\mathrm{pH} 2$ and at higher ionic strength. Both low $\mathrm{pH}$ and high ionic strength are expected to reduce electrostatic repulsion between the anionic species, the former by neutralizing the acid groups, the latter by screening through concentrated electrolyte. Hence, these data support the hypothesis that headgroup ionization can limit the adsorption density.

Interrogation of the thus-formed SAMS by examining the cyclic voltammetry of $4 \mathrm{mM} \mathrm{Fe}(\mathrm{CN})_{6}^{-4}$ also yielded supporting qualitative information. Figure 13 contrasts cyclic voltammetry data for MPA and MUA layers formed similarly to those in Figure $\mathbf{1 2 .}$ MPA layers (top trace) were not blocking to electron transfer between the Au disc electrode and diffusing $\mathrm{Fe}(\mathrm{CN})_{6}^{-4}$ ions, but MUA did strongly attenuate voltammetric currents, and hence, these currents are diagnostic of the permeability of the MUA layers. In these ex-situ experiments, performed on a mechanically polished Au disk electrode that was soaked in the indicated buffered thiol solutions, the following ordering in voltammetric currents were observed:

$1 \mathrm{mM} \mathrm{pH} 7>1 \mathrm{M} \mathrm{pH} 7>1 \mathrm{M} \mathrm{pH} 2 \approx 1 \mathrm{mM} \mathrm{pH} 2$

In other words, low ionic strength and high $\mathrm{pH}$ both correlate to permeable layers in this setting. This is fully consistent with the above SPR results. 


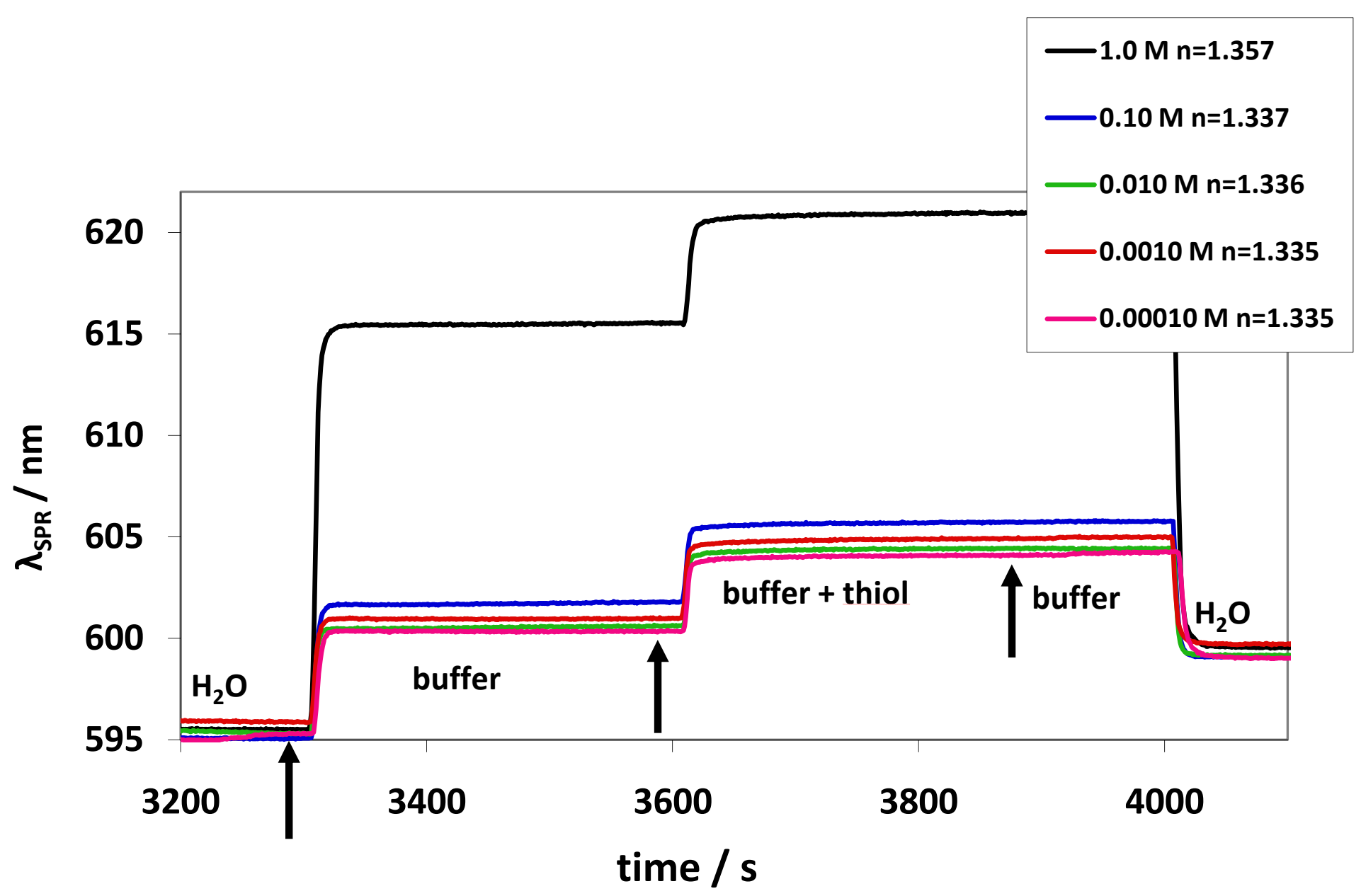

Figure 10. SPR sensograms reflecting chemisorption of 11-MUA anions ( $1.0 \mathbf{M}(A)$ to $0.00010 \mathbf{M}(E)$ in even decades) on gold surface from pH 7.2 phosphate buffer $->$ buffer + 11-MUA (first arrow) -> pH 7.2 phosphate buffer (second arrow) as a function of time (sec) vs. $\Delta \lambda_{S P R}(\mathrm{~nm})$. 


\subsection{Rationale for Binary SAM and Potentiostatic Deposition Experiments}

All of the above SPR data come with a significant and distressing caveat, however the reproducibility indicated by the error bars are good only for runs performed within the same day. For reasons that are unclear to us, the day-to-day variances were large enough to obscure the trends noted above and were stubbornly intransigent to our numerous quality control efforts along these lines. Alas, this fact does cast a degree of doubt on the above data and their interpretation. We feel that it is of value however to include the two-sigma within-day error bars. However, integration of, for example, similar MUA runs performed on a different day would likely mask the ordering observed in the above data because the magnitude of the SPR shifts from day-to-day were not reproducible. Our efforts have convinced us that it is well beyond the scope of this investigation to completely settle this issue.

Instead, and as a reaction to the above reproducibility issues, we adopted two additional strategies for better controlling the MUA surface coverage. Firstly, we adopted a strategy of providing a "diluent," neutral molecule MHX - that was intended to occupy void spaces between interacting MUA anion pairs and thus satisfy the available capacity of the Au surface for sulfur bonding but without incurring additional electrostatic cost. MHX incorporation was expected to substantially reduce MUA coverage because it provides a route to lower free energy ( $\mathrm{MHX}$ bonding) that does not involve MUA. Since MUA is approximately twice as long as MHX, we distinguish between MHX and MUA layers by the elevation in the SPR signal expected for full 
coverage monolayers of MUA, MHX or mixtures thereof. Mixed layer experiments begin in the next block (Phase 2). Here, we also implemented a higher $\mathrm{pH} 11$ condition for comparison but held the ionic strength of the phosphate buffer solution at $10^{-3} \mathrm{M}$. Secondly, we employed electrochemical (potentiostatic) control over the Au surface during the assembly process. This effort was mainly predicated on the reasoning that since the Au|electrolyte interface is charged, this charge may couple to the MUA anions and thus modulate the assembly process, but it enables as a fringe benefit the cathodic and anodic desorption of the alkanethiols thus obviating the ozonolysis cleaning step normally implemented between successive SAM formations. Potentiostatic experiments were implemented in Phases 3 and 4 below. 


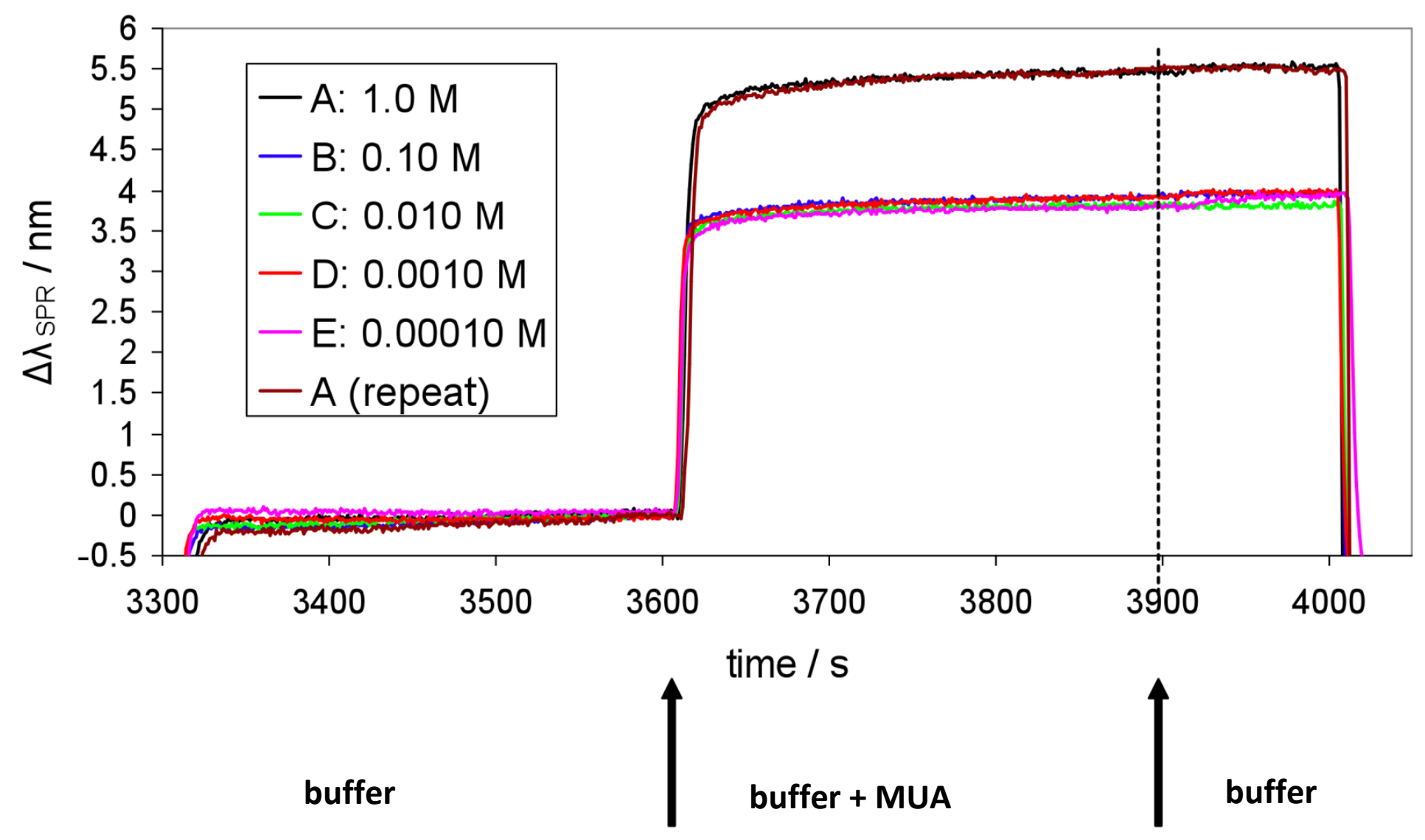

Figure 11. SPR sensogram reflecting chemisorption of 11-MUA anions (1.0 $\mathbf{M}(A)$ to $0.00010 \mathbf{M}(E)$ in even decades) on gold surface from pH 7.2 phosphate buffer -> buffer + 11-MUA (first arrow) -> pH 7.2 phosphate buffer (second arrow) as a function of time (sec) vs. $\Delta \lambda_{S P R}(\mathrm{~nm})$. 


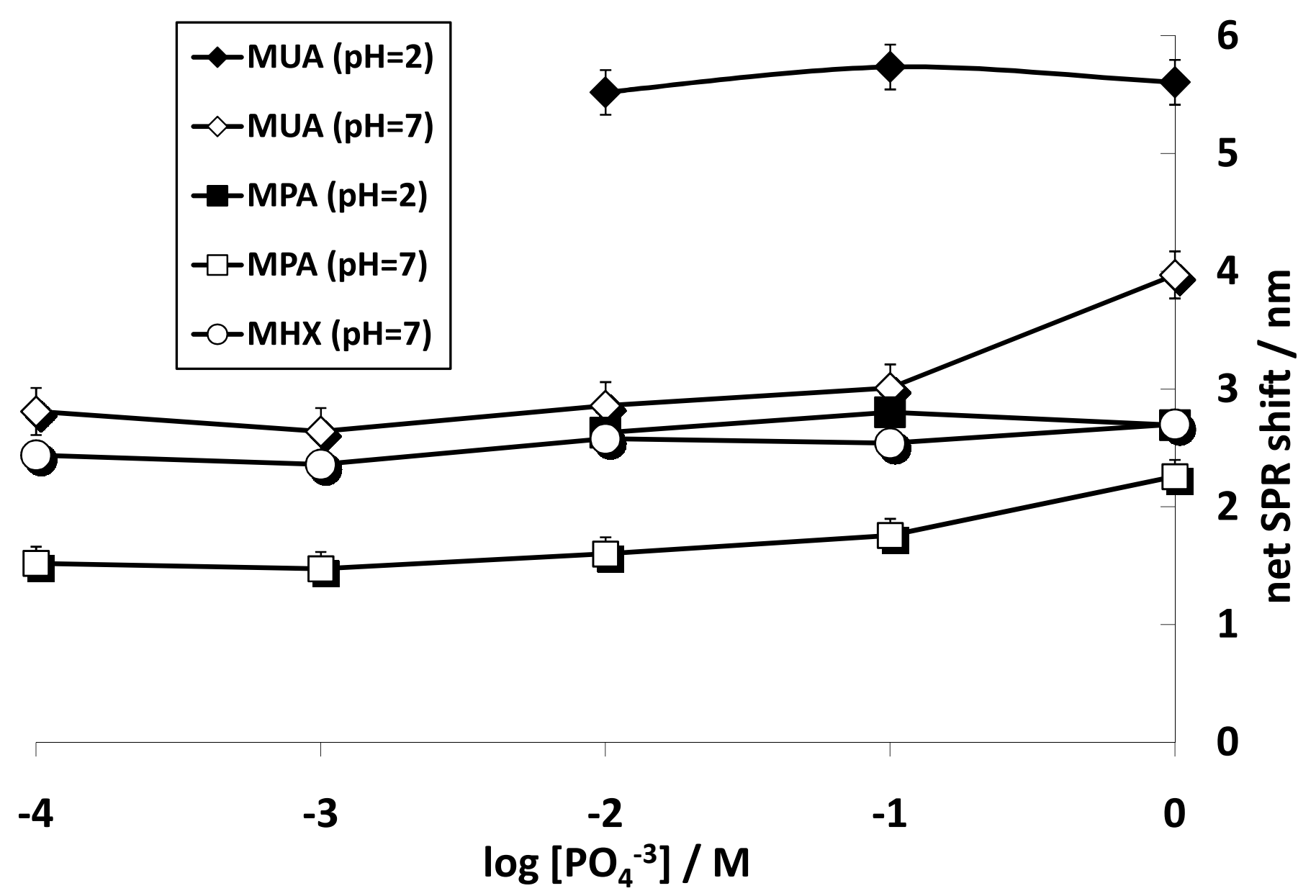

Figure 12. Net SPR shift results from replicate adsorptions of MUA and MPA from low and high pH buffers prepared at a series of increasing ionic strengths. Error bars are 2-s standard deviations for triplicate runs done on the same day. 


\section{Permeability of MUA layers to $\mathrm{Fe}(\mathrm{CN})_{6}{ }^{4-}$}

$1 \mathrm{M} \mathrm{KNO} 3 \mathrm{pH} 7,4 \mathrm{mM} \mathrm{K}_{4} \mathrm{Fe}(\mathrm{CN})_{6}, 20 \mathrm{mV} / \mathrm{s}$

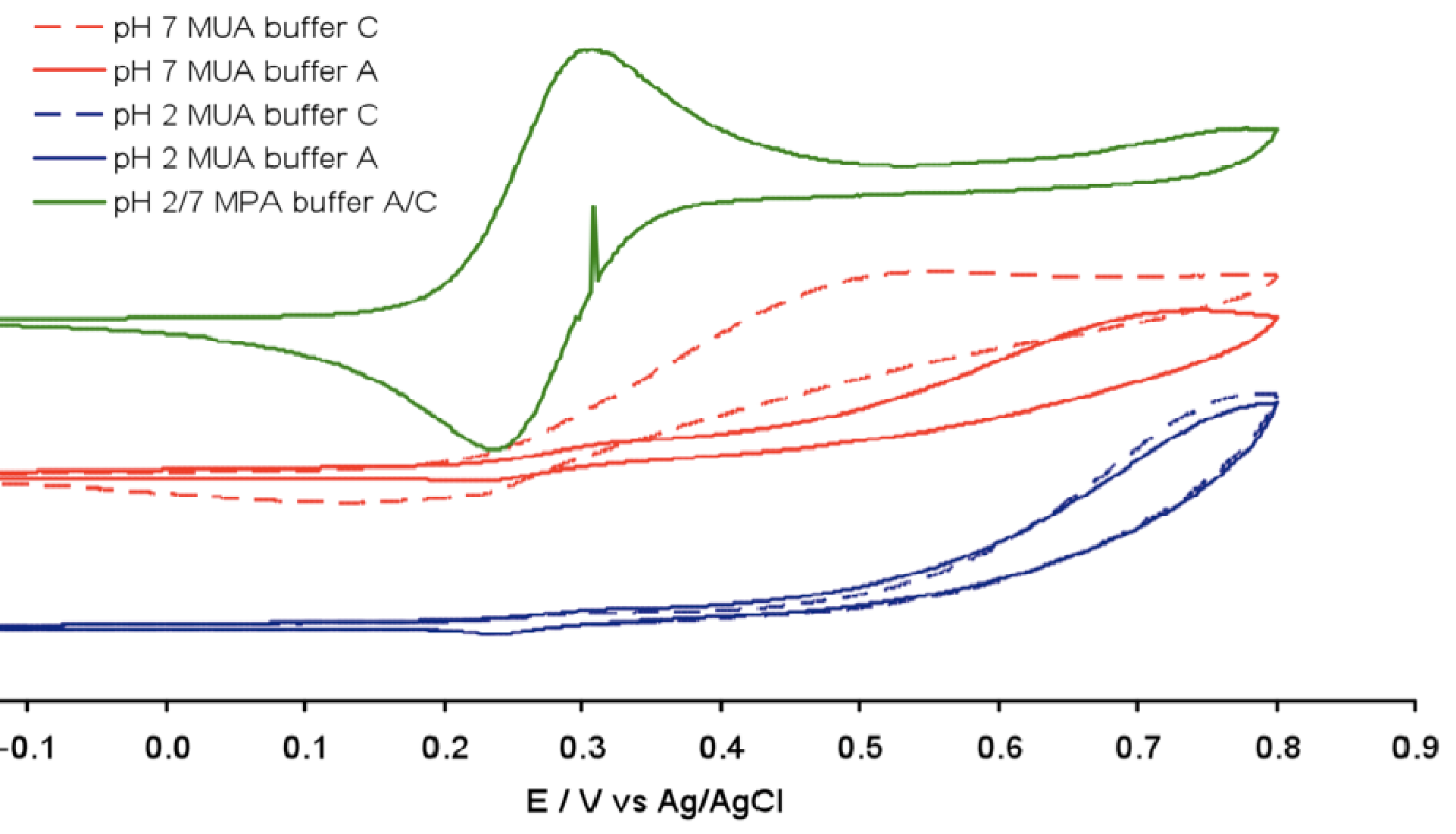

Figure 13. Cyclic voltammograms of $4 \mathrm{mM} \mathrm{Fe}(\mathrm{CN})_{6}^{-4}$ at $\mathrm{Au}$ (ex-situ, mechanically polished Au disk electrodes) coated with MUA (blue, $\mathrm{pH} 2$, and red, $\mathrm{pH} 7$ depositions) and MPA (green trace). 


\subsection{Phase 2: Open Circuit Measurements of the pH Dependence of Binary SAM Composition}

Experiments in this phase were carried out very similarly to those in Phase 1. Runs were performed with pure MUA, pure MHX or mixture of both MUA and MHX at

$\mathrm{pH} 3,7$ and 11 while the ionic strength was kept constant at $10^{-3} \mathbf{M}$. Specifically, we are interested in the phenomenon of MUA and MHX mixing in the SAM when both components are present in solution. Three cases can occur: 1.) MUA is favored 2.) MHX is favored, or 3.) Proportional binding. In other words, Au can choose to bind to either MUA or MHX, or have a mixture of both MUA and MHX. SPR sensogram results are shown in Figure 14. The left column corresponds to MHX, the middle to equimolar MUA-MHX and the right to MUA solutions. The top row is $\mathrm{pH} 3$, the middle $\mathrm{pH} 7$ and the bottom pH 11. The data are subject to some scatter, but a few trends are indicated. In general, the shifts get larger going from left to right as the solution incorporates MUA. This indicates that the mixed solutions are likely yielding mixed SAMs with intermediate average layer thicknesses. Qualitative differences are discernible as a function of $\mathrm{pH}$ for the case of pure MUA adsorption. From top to bottom in column 3, MUA adsorption sensograms are relatively well reproduced and show a qualitative difference between $\mathrm{pH} 3$ or 7 and 11. At pH 11, the adsorption transient is much more gradual but reaches essentially the same magnitude within the five minutes allotted to the transient. Our focus at this time is to assess the extent of monolayer mixing as a function of $\mathrm{pH}$. Figure 14 illustrates the net shifts after re-introduction of blank buffer 
for these triplicate analyses. The smaller, dark gray bars are MHX signals, the light gray is the mixed MUA/MHX and the white bars are for pure MUA. The left grouping is for $\mathrm{pH} 3$, the middle for $\mathrm{pH} 7$ and the right for $\mathrm{pH}$ 11. Average shifts for pure MHX (ca. 2 $\mathrm{nm}$ ) and for pure MUA (ca. $3.5 \mathrm{~nm}$ ) are well reproduced across the $\mathrm{pH}$ range and seem independent, on average, of $\mathrm{pH}$ within error. The mixed layers (light gray bars) all have average shifts intermediate between pure MUA and pure MHX indicating that these are mixed layers but the 2- $\sigma$ error bars overlap substantially and obscure any detail in possible $\mathrm{pH}$ effects on the mixing phenomenon. Our implicit assumption is that the signals recorded for these layers at 1950 s correspond to nearly full coverage SAMs. This is consistent both qualitatively with the saturation indicated by the shape of the adsorption transients and with the known structures of alkanethiol SAMS, i.e. that they form dense monolayers on gold with a high degree of structural order and attain sterically limited coverage corresponding to epitaxial $\sqrt{3} \times \sqrt{3 R 30}$ adlayer structures ${ }^{15}$. If this assumption is correct, i.e. that at $1950 \mathrm{~s}$ in Figure 14 the Au surface is at nearly unity coverage, then the magnitude of the SPR shift may be used to assess the total amount of MHX incorporated into mixed SAMs because of the known linearity between SPR shift and average SAM thickness. To the extent that this is the case, the bar graphs in Figure $\mathbf{1 5}$ may be interpreted as indicating the incorporation of $\mathrm{MHX}$ into mixed SAMs. 
MHX 3D

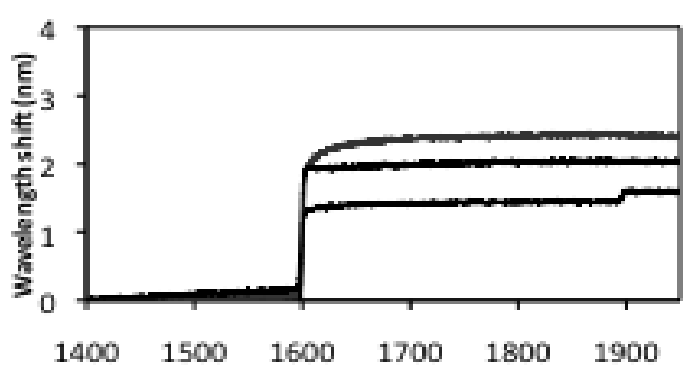

MHX 7D

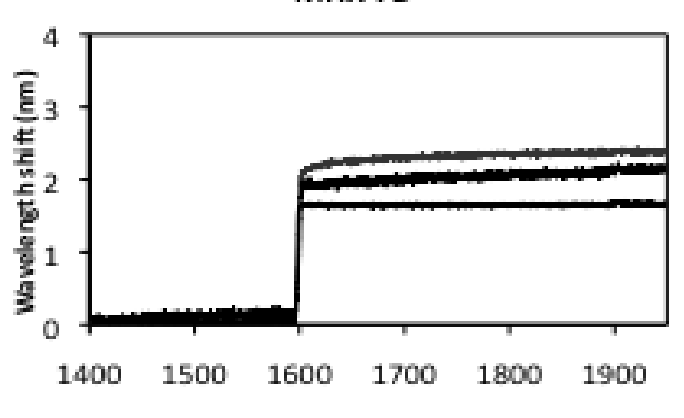

MHX 11D

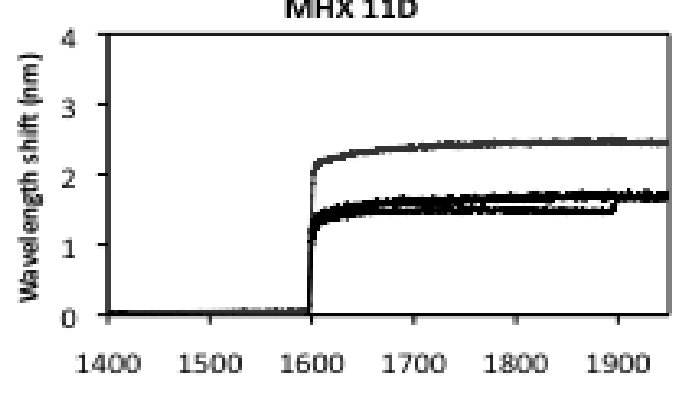

MUAMHX 3D

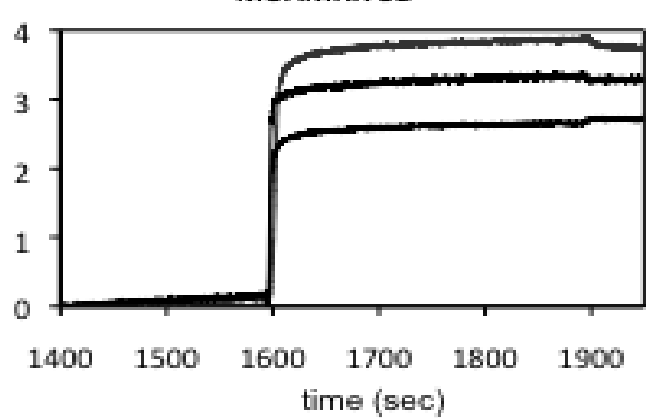

MUAMHX 7D

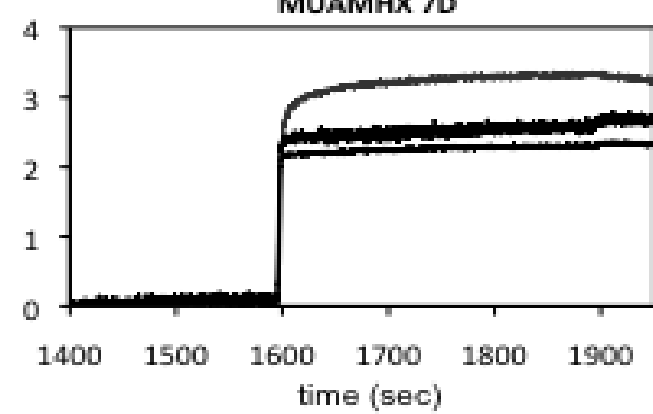

MUAMHX 11D

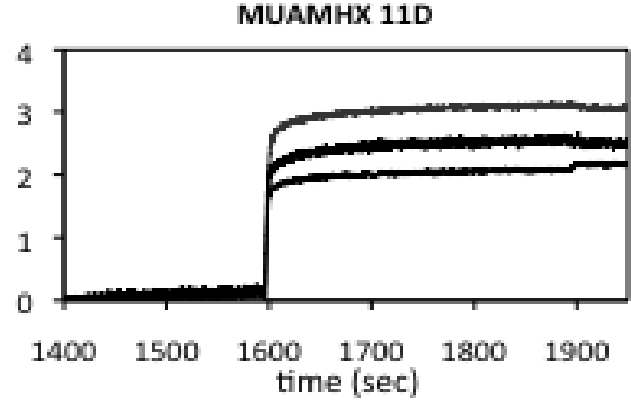

MUA 3D

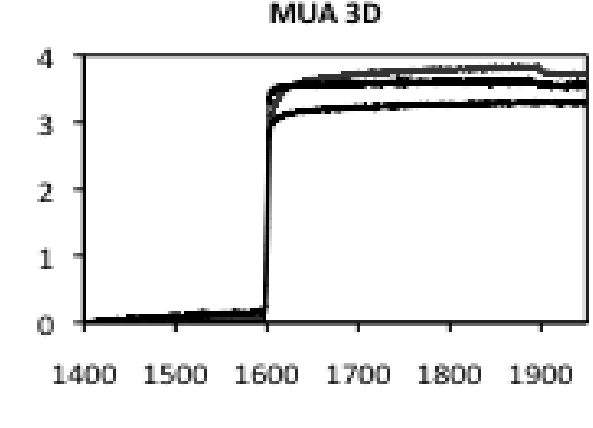

MUA 7D

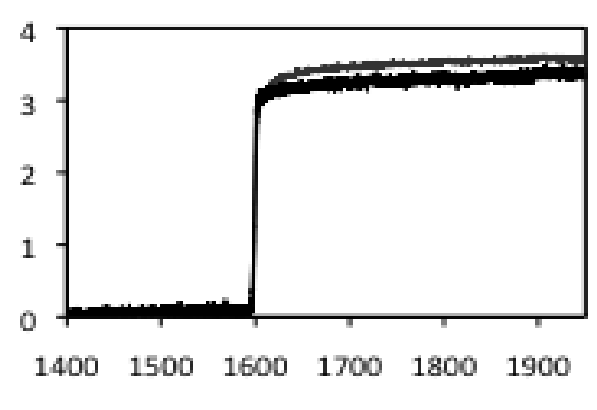

MUA 11D

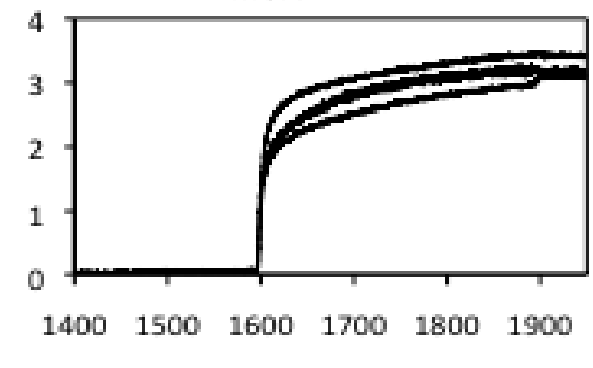

Figure 14. Open circuit SPR sensograms showing wavelength shift (nm) vs. time (sec) at $\mathrm{pH} 3,7$ and 11 for 11-MUA, 6-MHX, and mixed monolayer. 
A related issue is that binary SAMs of MUA and MHX may not be homogeneously mixed at the molecular level. There is every possibility that such SAMs may contain phase-separated domains of component ${ }^{12}$, i.e., MUA and MHX. Whitesides et al. ${ }^{14}$ studied the relationship between the composition of binary alkanethiol SAMs on gold as a function of the composition of the solutions from which they were formed. These studies suggested that monolayers tend to phase segregate - that is that they tend to form domains predominantly composed of either the long-chain or short-chain thiol constituents. A proper derivation of the relationship between the composition of the SAM and its solution constituents includes consideration of intermolecular interactions between components in the SAM. Both theory and experiment agree on a qualitative level that, in a two-component system of alkanthiolates on gold that is well equilibrated with alkanethiols in solution, a single phase is preferred at equilibrium.

The scatter evident in Figure $\mathbf{1 5}$ obscures the details of any assessment of the $\mathrm{pH}$ dependence of SAM mixing however. So, to try to improve this condition we explored potentiostatic deposition of the SAMs. That is, we poised the electrochemical potential of the Au SPR surface relative to the buffer solutions using a potentiostat. Monolayer assembly under these conditions is referred to as "closed circuit" or "potentiostatic" below. 


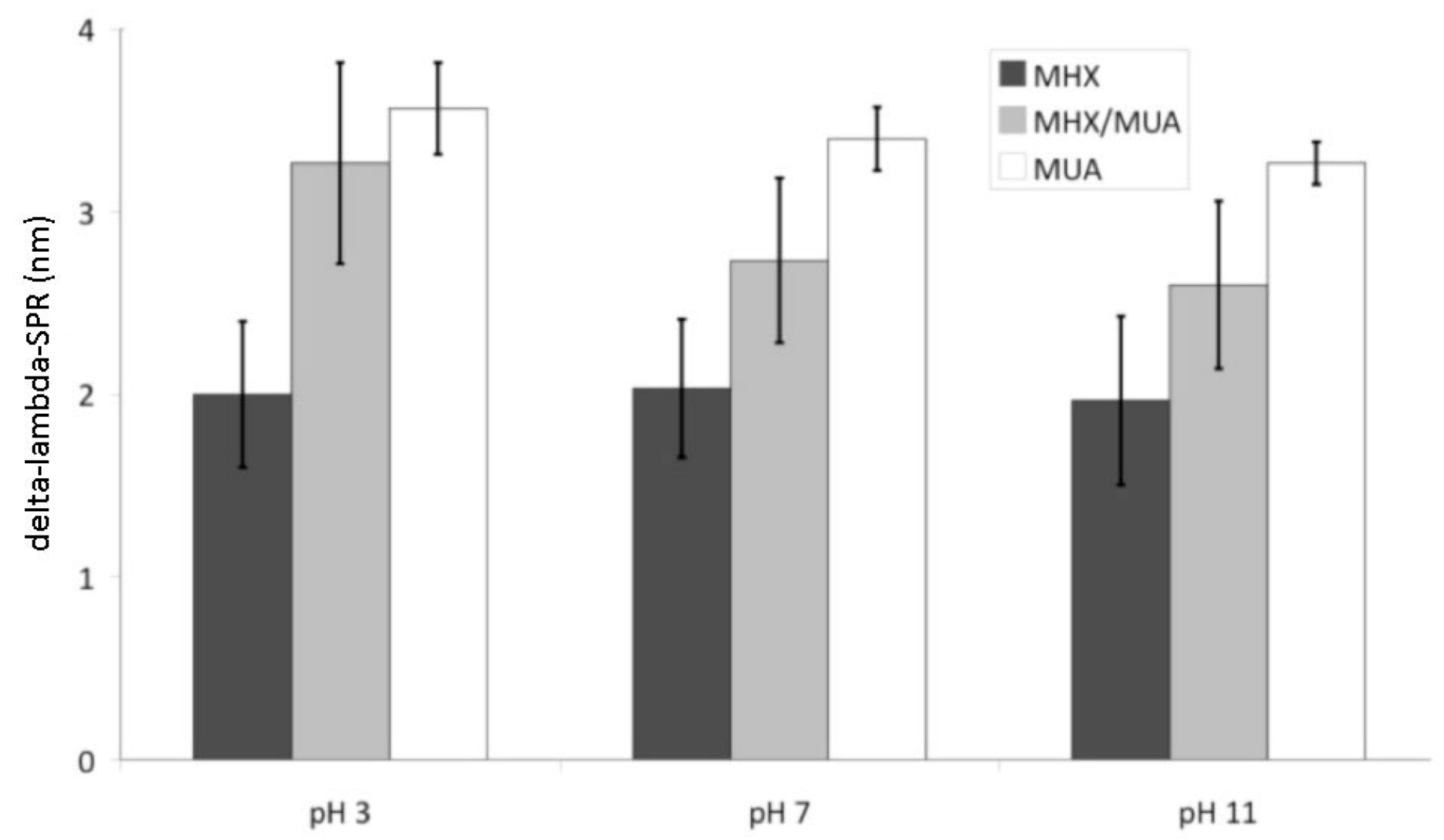

Figure 15. Open circuit bar graph showing delta-lambda-SPR $(\mathrm{nm})$ at $\mathrm{pH} 3,7$, and 11 for 11-MUA, 6-MHX and mixed monolayer. 


\subsection{Phase 3: Potential Dependence of Monolayer Assembly}

In this phase, a brief set of experiments was undertaken. Potentials (V vs.

$\mathrm{Ag} / \mathrm{AgCl}$ ) varying from $-0.50 \mathrm{~V}$ to $+0.50 \mathrm{~V}$ in increments of $0.25 \mathrm{~V}$ were applied successively to the Au sensor surface before and after the assembly of MUA at $\mathrm{pH} 7$, as

is shown in Figure 16. This plot illustrates the fact that the electrochemical potential of the clean Au substantially affects the baseline SPR wavelength, but that after assembly, this wavelength is much less potential dependent. This may be attributable to the refractive index contributions of the ions contained in the compact electrical double layer at the Au/water interface. Therefore, to properly understand the potential dependence of alkanethiol assembly using SPR, a correction needs to be made for displaced ions or whatever effect, unrelated to the thiol coating that is causing this baseline potential dependence. In this case, we use as a reference state the $0.00 \mathrm{~V}$ state and correct as follows: for the clean Au surface the SPR wavelength is recorded both at $0.00 \mathrm{~V}$ and the target potential 'E' - i.e. $\lambda_{0, C L E A N}$ and $\lambda_{E, C L E A N}$ are recorded. Then the potential is set to the desired value ' $\mathrm{E}$ ' and the assembly is conducted to completion. Then the two values $\lambda_{E, C O A T E D}$ and $\lambda_{0, \text { COATED }}$ are recorded. The SPR shift corrected to 0.00 V, $\Delta \lambda_{0}$ is then simply $\Delta \lambda_{0}=\lambda_{0, \text { COATED }}-\lambda_{0, \text { CLEAN. }}$ This is labeled as 'Corrected SPR Wavelength shift' in Figure 17. MUA-MHX, MUA and MHX are plotted as a function of net SPR wavelength $(\mathrm{nm})$ versus potential $(\mathrm{V})$ in Figure 17. Note in Figure 17 at potentials positive of $0.00 \mathrm{~V}$, the corrected $\Delta \lambda_{S P R}$ values are significantly higher than those compared to negative and neutral potential, and particularly in the case of $\mathrm{MHX}$ 
containing solutions the potentiostatic assembly curves had a tendency to not level off, i.e. to continue to shift to quite large $\Delta \lambda_{\text {SPR }}$ values and for a long time. The reason for these 'runaway' signals is unclear, but we speculate that there may be some sulfur oxidation process occurring that gives us this phenomenon. Hence potentiostatic deposition at potentials positive of $0.00 \mathrm{~V}$ were subsequently avoided. The limited set of data in Figure 17 allowed us to define a useable potential range for subsequent experiments. But due to the limited number of trials conducted at each potential, the details of SAM mixing cannot be assessed from this exploratory analysis. However, it was clear that reasonable adsorption transients could be acquired at electrochemical potentials between -0.50 and $0.00 \mathrm{~V}$ vs. $\mathrm{Ag} / \mathrm{AgCl}$. Notably, clean Au typically exhibited an open circuit potential near $-0.1 \mathrm{~V}$ vs. our $\mathrm{Ag} / \mathrm{AgCl}$ reference electrode, but this does not necessarily mean that at $0.00 \mathrm{~V}$ the surface is above the potential of zero charge i.e. the surface charge state of the Au is still not known and may be positive, neutral or negative at $0.00 \mathrm{~V}$. 


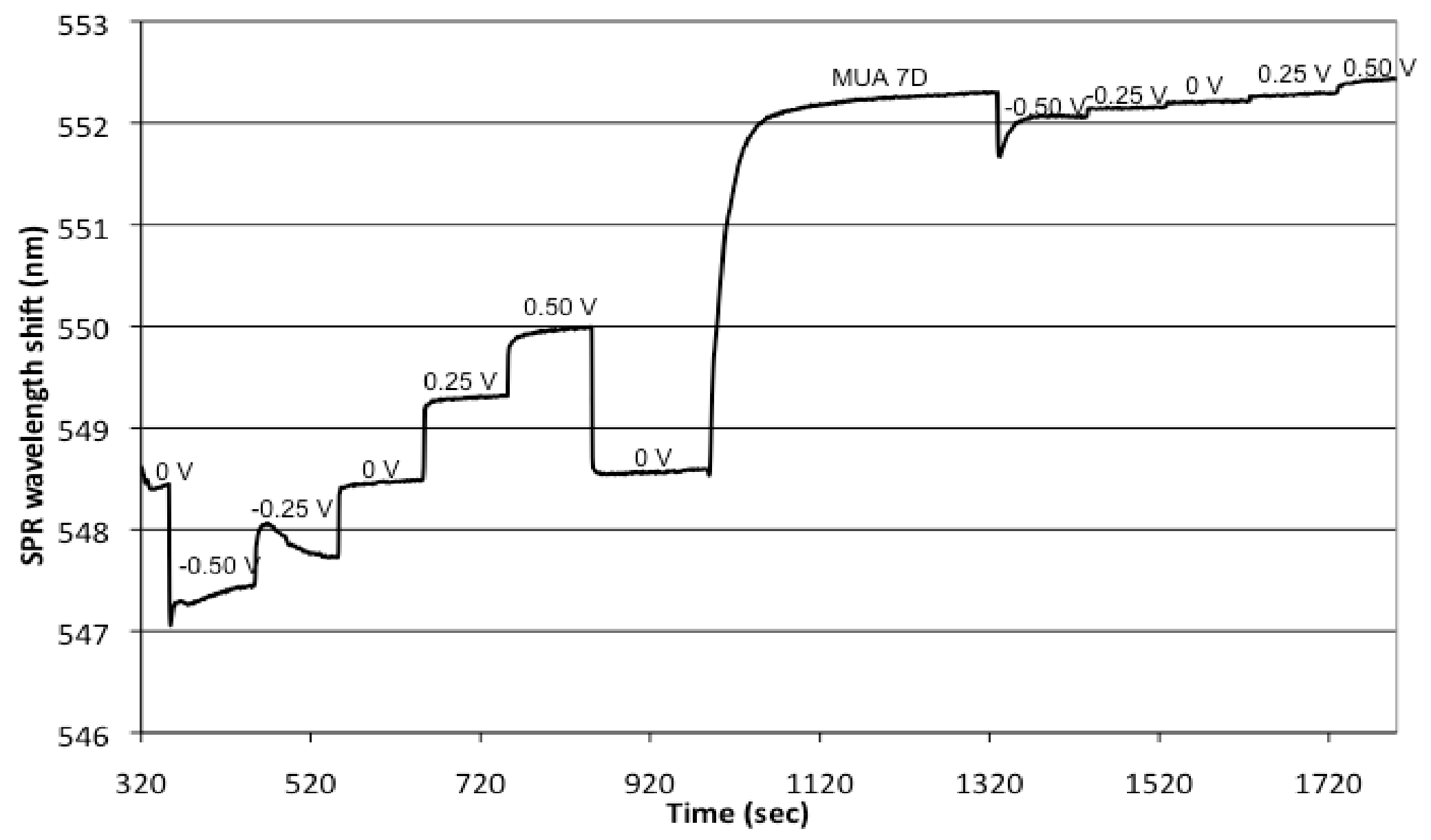

Figure 16. Potentiostatic dependence experiment illustrating the potential dependence of the SPR wavelength as the potential is incremented from $-0.50 \mathrm{~V}$ to $+0.50 \mathrm{~V}$ before and after assembly of MUA 7D on gold surface. 
In the next phase of experiments, we chose to investigate the effect of $\mathrm{pH}$ on the assembly of binary SAMs on Au in a closed circuit environment where the potential was maintained at $0.00 \mathrm{~V}$ vs. $\mathrm{Ag} / \mathrm{AgCl}$ - a potential chosen arbitrarily and close to the open circuit potential. This leads us into the next phase of experiments described in Phase 4 of Table 1 where potential-controlled assembly of MUA, MUA-MHX and MHX was implemented at $\mathrm{pH} 3,7$ and 11 while the ionic strength of the phosphate buffer was kept at $10^{-3} \mathrm{M}$ and the potential held at $0.00 \mathrm{~V}$.

\subsection{Phase 4: Closed Circuit Measurements of the pH Dependence of Binary SAM Composition.}

As described above, in Phase 4 experiments were undertaken similarly to those in Phase 2 but in a closed circuit setting. This means that during the assembly of MUA, SPR sensogram results shown in Figure 18 yielded the $\Delta \lambda_{\text {SPR }}$ values that were much more reproducible than those recorded at open circuit. Compared to the open circuit case, the transients acquired seem to have a more gradual quality especially for the case of MUA. The reason for this is unclear. The improvement in reproducibility is illustrated in Figure 19. The 2- $\sigma$ error bars are quite small in this case and allow us to conclude, again assuming unity coverage as above, that at $0.00 \mathrm{~V}$, SAM mixing only occurs at $\boldsymbol{p H}$ 3; whereas, in pH 7 and 11 cases, the binary SAM comprises almost exclusively MHX. This surprising result appears to reflect a strong preference for the neutral thiol components when the assembly is conducted potentiostatically at $0.00 \mathrm{~V}$ vs. $\mathrm{Ag} / \mathrm{AgCl}$. Only at $\mathrm{pH} 3$, where solution-phase MUA is predominantly protonated and therefore 
neutral, does it incorporate into mixed MUA/MHX monolayers. It is possible that the potential of zero charge for Au under these conditions is far positive of $0.00 \mathrm{~V}$, and hence anionic MUA is being selectively repulsed from the interfacial region at this potential. However, assuming this to be the case, the fact that we typically observe an open circuit potential of $-0.1 \mathrm{~V}$, it would suggest that at open circuit adsorptions the same rejection of MUA might also predominate. This was clearly not the case however.

From the perspective of preparing suitable SPR anchor layers however this result is highly significant. In such an anchor layer, the MUA carboxylate end groups serve as sites where longer-chain molecules can be covalently coupled. The desirable anchor layer will have a limited number of MUA components separated by MHX in such a way as to preserve a significant amount of lateral spacing for the long-chain constituents of the desired NEM active adlayer. So, using this study as a guide, we can conclude that potentiostatic assembly held at $0.00 \mathrm{~V}$ and $\mathrm{pH} 3$ is likely to reproducibly incorporate about 50\% MUA into binary mixed SAMs and may be optimal for achieving our longterm goal of creating NEM active layers on the Au surface. 


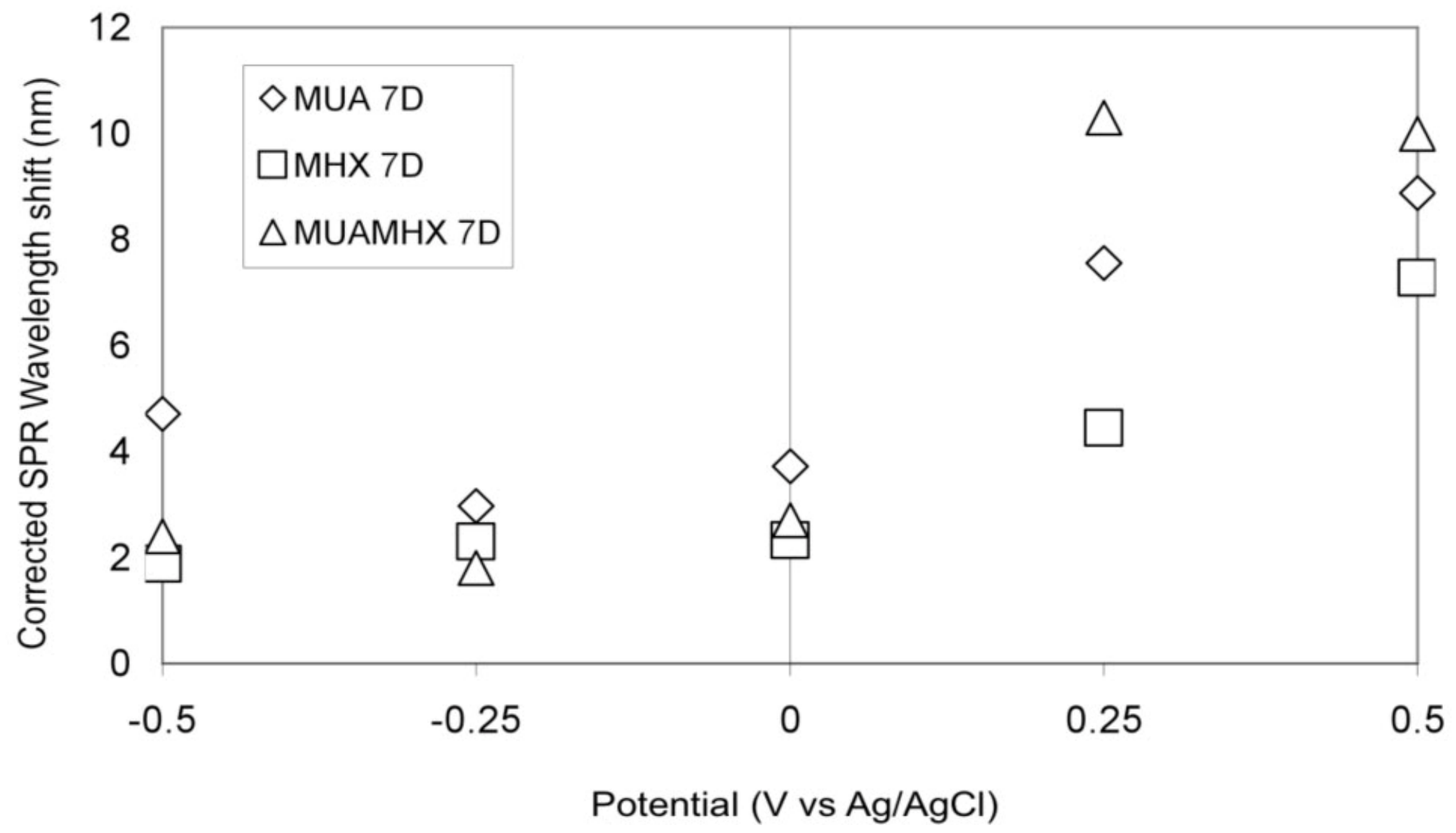

Figure 17. Potential dependence plot of MUA, MUAMHX and MHX as a function of Potential (V vs. Ag/AgCl) vs. Corrected SPR Wavelength shift $(\mathrm{nm})$ on gold surface at $\mathrm{pH}$ 7.MUA/MHX and $\mathrm{MHX}$, a potential of $0.00 \mathrm{~V}$ was applied to the Au sensor surface. 

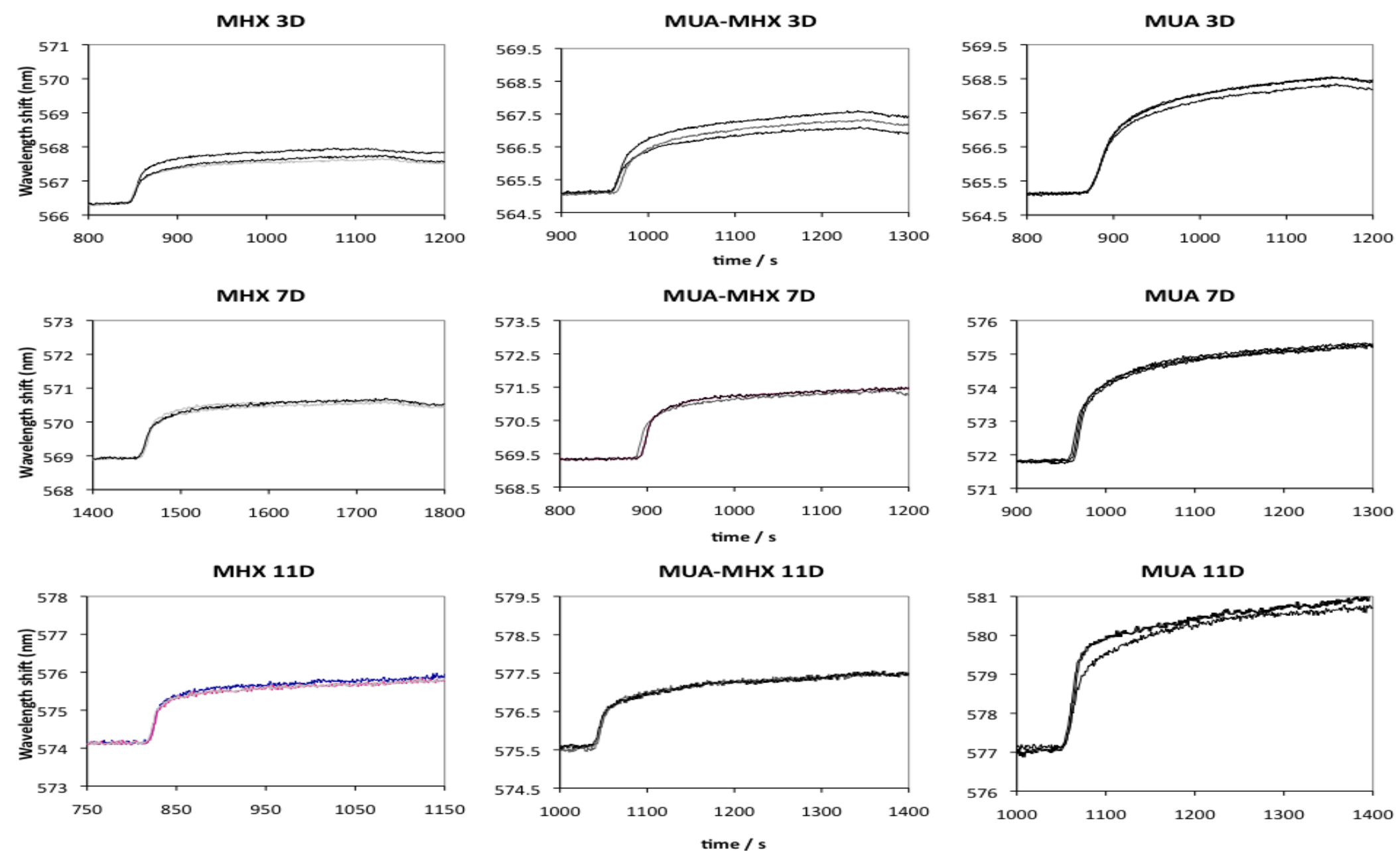

Figure 18. Closed circuit SPR sensograms showing wavelength shift $(\mathrm{nm})$ vs. time $(\mathrm{sec})$ at $\mathrm{pH} 3,7$, and 11 for 11-MUA, 6-MHX, and mixed monolayer with $\mathrm{E}=0.00 \mathrm{~V}$. 


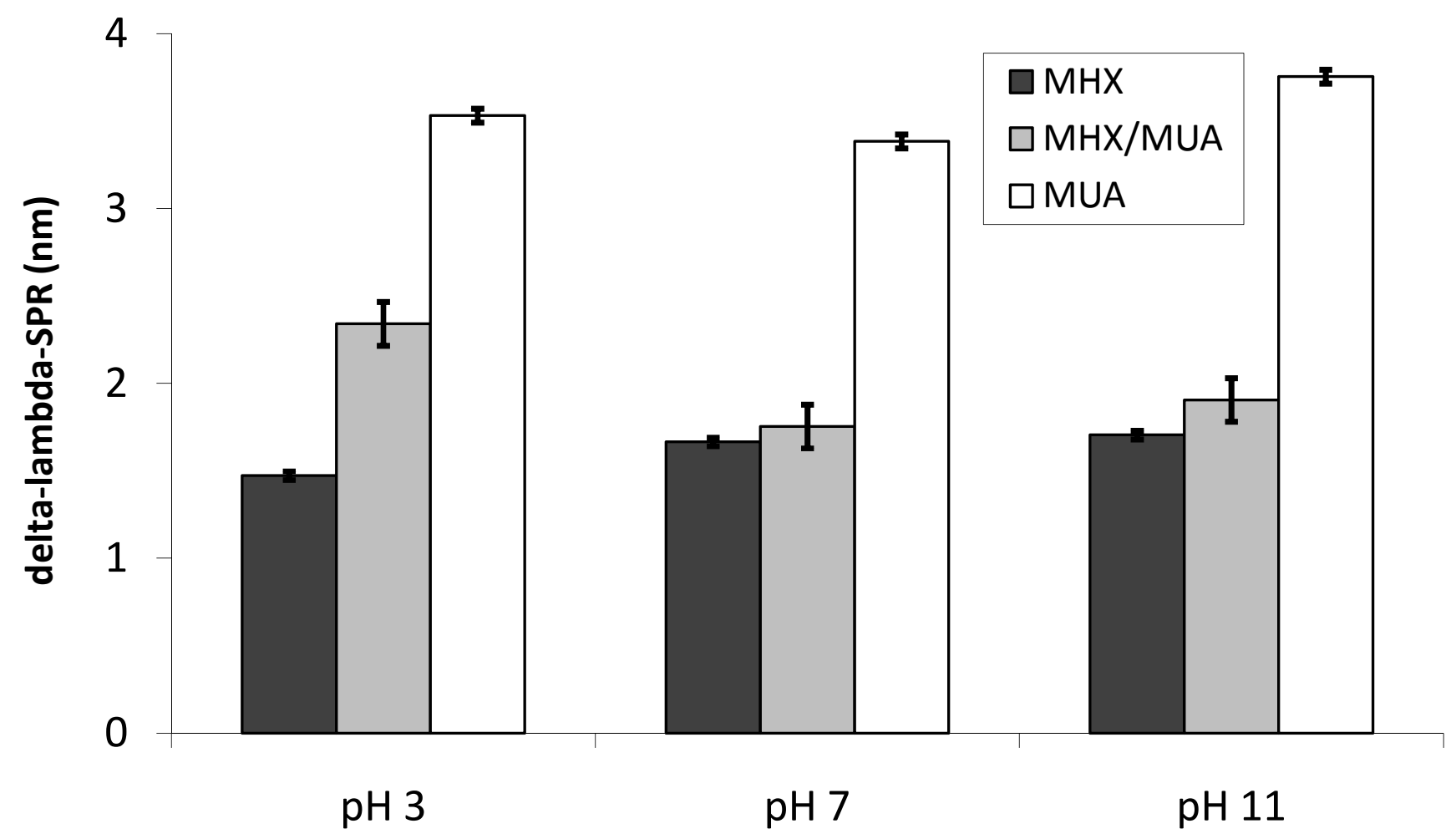

Figure 19. Closed circuit bar graph showing delta-lambda-SPR (nm) at $\mathrm{pH} 3,7$ and 11 for 11-MUA, 6-MHX and mixed monolayer with $\mathrm{E}=0.00 \mathrm{~V}$. 


\section{CONCLUSION}

Acid terminated alkanethiol SAM formation from phosphate buffer solutions onto a Au SPR sensor surface was described in this thesis. Initial experiments utilizing varying ionic strengths of phosphate buffer solutions from $0.1 \mathrm{mM}$ to $1 \mathrm{M}$ supported our initial hypothesis that, at $\mathrm{pH} 7$, electrostatic repulsion between the anionic carboxylate groups would limit the density of MUA layers formed from this solution. However, reproducibility problems clouded our confidence in this approach for making lowdensity MUA layers. To solve this problem, a diluent molecule, MHX and an alternative approach to the SAM deposition, i.e. deposition under electrochemical potential control, was implemented in electrochemical-SPR experiments. We found that potentiostatic assembly dramatically improved the reproducibility of the SAM formation results. Surprisingly, we also found that only at $\mathrm{pH} 3$ was MUA incorporated into binary mixed SAMs, and at roughly $50 \%$ surface incorporation from a $50 \%$ solution composition. Using this as a guide, we believe we are on a sound footing for the next phase of our long-term project goal of creating NEM active layers on the Au surface. The dilute MUA-MHX layers formed at $\mathrm{pH} 3$ as above appear to present a fractional coverage of MUA proportional to solution composition, and, provided this behavior holds for other solution compositions, this will allow us to vary the density of the carboxylate anchors in future work where the formation of amide-coupled oligomeric species is undertaken. 


\section{FUTURE WORK}

In order to achieve our long-term goal of creating NEM arms that have an electro-mechanical response to electrochemical potential, we need to be able to tether molecules onto the existing carboxylate anchor layer. Presently, we plan to use a 3000 MW polyethyleneglycol (PEG) oligomer that is terminated on one end with a carboxylate and the other end with a protected amine (SunBright Chemical Company, Japan). This may then serve as a linker arm for attaching the quantum dot onto the binary SAM.

But because of the cost of this molecule, we have chosen to do exploratory experiments making amide bonds to MUA layers and assessing the success of these studies. To this end, we have begun a series of in-situ coupling experiments wherein coupling is monitored with SPR. These experiments that were carried out on MUA layers prepared as above (MUA/MHX pH 3, 0.00V vs Ag/AgCl) and using 1,4phenylenediamine as a model amine in conjunction with coupling agents such as Nhydroxylsuccinimide (NHS) and 1-(3-dimethylamino)propyl)-3-ethylcarbodiimide hydrochloride (EDC). Initial results yielded a puzzling response suggesting that longer reaction times may be needed. These and similar experiments represent the beginning of the next phase of this project - covalent attachment of NEM linker arms using amide chemistry. 


\section{REFERENCES}

(1) Hu, W.P.; Chen, S.J.; Huang, K.T.; Hsu, J.H.; Chen, W.Y.; Chang, G.L.; Lai, K.A. Biosensors and Bioelectronics 2004, 19, 1465 - 1471.

(2) Liedberg, B.; Nylander, C.; Lundstrom, I. Sensors and Actuators B: Chemical 1983, 4, 299.

(3) Bain, C. D.; Whitesides, G. M. Advanced Materials 1989, 1, 110.

(4) Love, J. C.; Estroff, L. A.; Kriebel, J. K.; Nuzzo, R. G.; Whitesides, G. M. Chemical Reviews 2005, 105, 1103-1170.

(5) Mrksich, M.; Grunwell, J. R.; Whitesides, G. M. Journal of the American Chemical Society 1995, 117, 12009-12010.

(6) Bain, C. D.; Troughton, E. B.; Tao, Y. T.; Evall, J.; Whitesides, G. M.; Nuzzo, R. G. Journal of the American Chemical Society. 1989, 111, 321-335.

(7) Chen, S.; Li, L.; Boozer, C. L.; Jiang, S. The Journal of Physical Chemistry B 2001, 105, 2975-2980.

(8) Diao, P.; Hou, Q.; Guo, M.; Xiang, M.; Zhang, Q. Journal of Electroanalytical Chemistry 2006, 597, 103-110.

(9) Arakawa, T.; Hobara, D.; Yamamoto, M.; Kakiuchi, T. Electrochemistry Communications 2005, 7, 848-852.

(10) Widrig, C.; Chung, C.; Porter, M. D. Journal of Electroanalytical Chemistry. 1991, $310,335$.

(11) Calvente, J. J.; Kovacova, Z.; Sanchez, M. D.; Andreu, R.; Fawcett, W. R. Langmuir, 1996, 12, 5696.

(12) Loglio, F.; Schweizer, M.; Kolb, D. M. Langmuir 2003, 19, 830.

(13) Whitesides, G.M; Bain, C.D.; Evall, J. Journal of the American Chemical Society 1989, 111, 7155-7164.

(14) Porter, M.D.; Alves, C.A.; Smith, E.L. Journal of the American Chemical Society 1992, $114,1222-1227$. 